\title{
Drawing of micro-structured fibres: circular and non-circular tubes
}

\author{
Yvonne M. Stokes ${ }^{1, \dagger}$, Peter Buchak ${ }^{2}$, Darren G. Crowdy ${ }^{2}$ and \\ Heike Ebendorff-Heidepriem ${ }^{3}$ \\ ${ }^{1}$ School of Mathematical Sciences, The University of Adelaide, North Terrace, Adelaide, \\ SA 5005, Australia \\ ${ }^{2}$ Department of Mathematics, Imperial College London, 180 Queen's Gate, London SW7 2AZ, UK \\ ${ }^{3}$ Institute for Photonics and Advanced Sensing, School of Chemistry and Physics, \\ The University of Adelaide, North Terrace, Adelaide, SA 5005, Australia
}

(Received 21 January 2014; revised 8 May 2014; accepted 11 July 2014; first published online 14 August 2014)

A general mathematical framework is presented for modelling the pulling of optical glass fibres in a draw tower. The only modelling assumption is that the fibres are slender; cross-sections along the fibre can have general shape, including the possibility of multiple holes or channels. A key result is to demonstrate how a so-called reduced time variable $\tau$ serves as a natural parameter in describing how an axial-stretching problem interacts with the evolution of a general surface-tension-driven transverse flow via a single important function of $\tau$, herein denoted by $H(\tau)$, derived from the total rescaled cross-plane perimeter. For any given preform geometry, this function $H(\tau)$ may be used to calculate the tension required to produce a given fibre geometry, assuming only that the surface tension is known. Of principal practical interest in applications is the 'inverse problem' of determining the initial cross-sectional geometry, and experimental draw parameters, necessary to draw a desired final cross-section. Two case studies involving annular tubes are presented in detail: one involves a cross-section comprising an annular concatenation of sintering near-circular discs, the cross-section of the other is a concentric annulus. These two examples allow us to exemplify and explore two features of the general inverse problem. One is the question of the uniqueness of solutions for a given set of experimental parameters, the other concerns the inherent ill-posedness of the inverse problem. Based on these examples we also give an experimental validation of the general model and discuss some experimental matters, such as buckling and stability. The ramifications for modelling the drawing of fibres with more complicated geometries, and multiple channels, are discussed.

Key words: capillary flows, low-Reynolds-number flows, lubrication theory

\section{Introduction}

Microstructured optical fibres (MOFs) have revolutionised optical fibre technology, promising a virtually limitless range of fibre designs for a wide range of applications

$\dagger$ Email address for correspondence: yvonne.stokes@adelaide.edu.au 
(Knight 2003; Monro \& Ebendorff-Heidepriem 2006). An array of air channels running along the length of a fibre, with diameters comparable to the wavelength of light, provide the necessary spatial change in the refractive index to guide light along the core. These fibres are fabricated as depicted in figure 1; a preform $(1-3 \mathrm{~cm}$ diameter and with a length measured in tens of centimetres) with appropriate geometry is fed into a heated region at a feed speed $U_{0}$ and pulled at a higher speed $U_{L}$ by winding onto a spool some distance downstream beyond the neck-down region, comparable to but not necessarily identical with the heated region. Internal channels may be pressurised. The resulting fibre may have a diameter of less than a millimetre and a length of a kilometre or more, while the internal air channels have microor even nano-scale diameters. From the start to the end of the neck-down region, this fibre drawing process modifies both the scale and shape of the cross-sectional geometry, so that achieving a desired fibre design is non-trivial, since the required preform geometry is not just the fibre geometry at a larger scale.

In this paper we describe a mathematical model for the drawing of MOFs, assuming that inertial effects are negligible and that there is no pressurisation of internal channels. The model may be solved, given an initial preform geometry and appropriate draw parameters, to give the final fibre geometry: the 'forward problem'. In principle, the model can also be used to determine a preform geometry and draw parameters that will yield a desired fibre design: the 'inverse problem'. Solution of both forward and inverse problems will be demonstrated for tubular fibres that may not be axisymmetric, and extension to more general fibre geometries will be discussed. We aim to improve understanding of the parameters that control the practical achievability of a desired air-solid geometrical structure in the final fibre. The model has application to drawing of all preforms, extruded, drilled and comprised of an assembly of stacked capillary tubes (Monro \& Ebendorff-Heidepriem 2006).

Modelling of fibre drawing was first motivated by the spinning of synthetic fibres for textiles and an early detailed one-dimensional model for this, obtained by an asymptotic expansion in terms of the small aspect ratio of the fibre, was presented by Matovich \& Pearson (1969). This model was derived in an Eulerian reference frame, in which fibre drawing is a steady-state problem. Yarin, Gospodinov \& Roussinov (1994) and Fitt et al. (2002), motivated by the drawing of optical fibres, derived Eulerian one-dimensional models for drawing of circular tubes and made some progress in quantifying the effects of inertia, gravity and surface tension. However, of particular relevance here, they did not obtain a complete exact solution for the case of drawing a circular-tube fibre with neglect of internal hole pressurisation, gravity, inertia and temperature variation.

Lagrangian coordinate systems have, quite commonly, been used for models of extensional flows; see, for example, Wilson (1988), Kaye (1990), Yarin (1993) and Stokes, Tuck \& Schwartz (2000). Our work here builds directly on the work of DeWynne, Howell \& Wilmott (1994), Cummings \& Howell (1999) and Griffiths \& Howell (2007, 2008), all of whom used an asymptotic approach and a Lagrangian reference frame, following a cross-section through the neck-down region. In this reference frame fibre drawing is an unsteady problem. A detailed derivation of the equations governing the evolution of a non-axisymmetric slender Newtonian fibre, containing no holes in the cross-section, in the case where surface tension has negligible effect, was given in DeWynne et al. (1994). There it was shown that, to leading order, in the Lagrangian reference frame travelling with a cross-section from $x=0$ to $x=L$ (see figure 1), the cross-section changes in size but otherwise retains its initial shape. (It is usual to assume that the neck-down length $L$ is well 


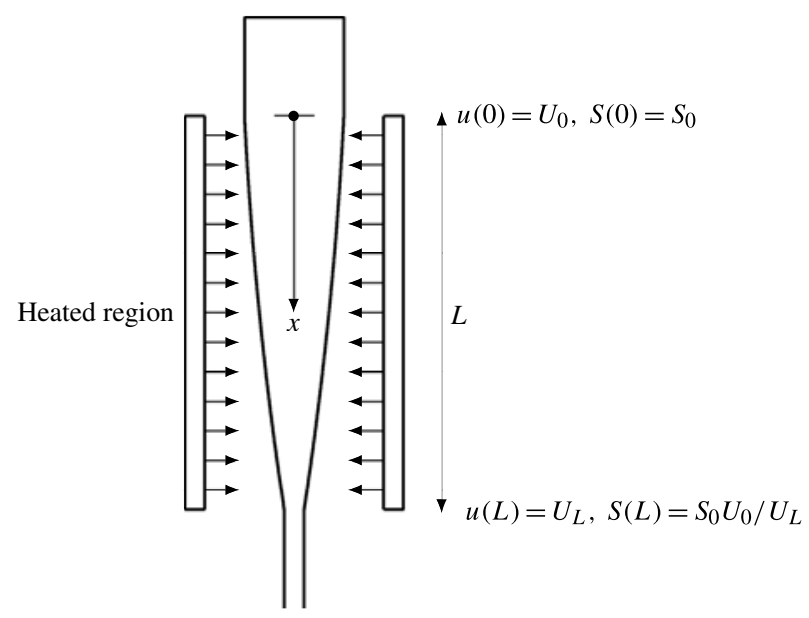

FIgURE 1. Drawing of a viscous fluid fibre. At $x=0$ the cross-sectional geometry is prescribed and has area $S_{0}$, while the fluid 'feed speed' is $U_{0}$; at $x=L$ the fluid velocity, or 'draw speed', is $U_{L}>U_{0}$. We suppose that for $x>L$ there is no more axial stretching or change in the cross-sectional geometry.

approximated by the length of the heated zone of the draw tower.) Cummings \& Howell (1999) considered the case where surface tension is non-negligible and the cross-section (which contains no holes) undergoes changes in size and shape. They showed, again using a Lagrangian reference frame and, importantly, a particular reduced time variable $\tau$, that the evolution of the shape of the cross-section reduces to a classical two-dimensional Stokes flow free-boundary problem driven by unit surface tension, which we shall refer to as the transverse-flow problem, and that this is coupled to a one-dimensional axial-stretching problem, which determines the size of the cross-section over time; the coupling of the two-dimensional and one-dimensional models is through the total length of the cross-sectional perimeter boundary as a function of reduced time. Cummings \& Howell (1999) do not describe their implementation of the coupling in any detail. Griffiths \& Howell $(2007,2008)$ built on this approach when they considered the cross-sectional shape of slender thin-walled viscous tubes, subject to deformations due to both stretching and surface tension. They used the Lagrangian reference frame and the reduced time variable $\tau$ introduced by Cummings \& Howell (1999) for the transverse-flow problem, and the Eulerian reference frame for the axial-stretching problem, and, for isothermal conditions, obtained an explicit asymptotic solution for a general initial cross-sectional shape in the slow flow limit (when inertial effects are negligible). This is a remarkable result.

In this paper we show that the result of Griffiths \& Howell $(2007,2008)$ is a very special case of a much more general mathematical structure that exists for the fibre-drawing problem. Our main result is to show that it is possible to obtain explicit solutions to the fibre-drawing problem for arbitrary cross-plane profiles, without the success of the formulation relying on any special assumptions about the cross-plane geometry. By writing and solving the axial-stretching problem, as well as the transverse-flow problem, in the Lagrangian reference frame, with the reduced time $\tau$ as the independent variable, we find that the solution to the axial-stretching problem can be written explicitly for any cross-sectional geometry in terms of the 
single function

$$
H(\tau)=\exp \left(-\frac{1}{12} \int_{0}^{\tau} \tilde{\Gamma}\left(\tau^{\prime}\right) \mathrm{d} \tau^{\prime}\right),
$$

where $\tilde{\Gamma}$ is the total dimensionless cross-sectional perimeter calculated from a suitably rescaled transverse-flow problem. From knowledge of this function $H(\tau)$, it turns out that the Eulerian axial coordinate $x$, the axial velocity $u$, and the cross-sectional area $S$ are all given by explicit formulae with $\tau$ serving as the natural parameter. For example, in the constant-viscosity (isothermal) case we find

$$
\begin{gathered}
S(\tau)=\frac{1}{H(\tau)^{2}}\left(1-\frac{\sigma^{*}}{\gamma^{*}} \int_{0}^{\tau} H\left(\tau^{\prime}\right) \mathrm{d} \tau^{\prime}\right)^{2}, \\
u(\tau)=\frac{1}{S(\tau)}, \\
x(\tau)=-\frac{1}{\sigma^{*}} \log (H(\tau) \sqrt{S(\tau)}) .
\end{gathered}
$$

A viscosity that depends on axial position can also be handled by appropriate scaling. Here, $\gamma^{*}$ is the scaled surface tension and $\sigma^{*}$ is the scaled tension in the fibre, which we call the fibre tension. It is seen that the coupling between the transverse-flow problem and the axial-stretching problem is solely through the function $H(\tau)$ and so that function completely determines the three-dimensional geometry. Furthermore, the preform and fibre geometries and physical surface tension determine the fibre tension, without needing to know the viscosity profile or neck-down length $L$. A similar observation was made in a more restricted context by Chen \& Birks (2013).

Linear stability analyses of the isothermal drawing of circular-rod and circular-tube fibres has shown that draw-resonance instability occurs at a constant draw ratio in excess of a little more than 20 (Pearson \& Matovich 1969; Yarin et al. 1994; Fitt et al. 2002) and this has been experimentally confirmed (Denn 1980). Nevertheless, in practical fibre drawing, where there is control of the fibre tension and an axial temperature gradient, fibres with simple through to complex structure are drawn at large draw ratios up to 10000 , so that control of fibre tension, cooling of the fibre and, perhaps, other factors appear to have a stabilising effect (Pearson \& Matovich 1969; Gospodinov \& Yarin 1997; Scheid et al. 2010). Therefore, although the occurrence of draw resonance at draw ratios in excess of 20 cannot be precluded, we leave this matter for future investigation and here allow draw ratios well in excess of the upper limit suggested by linear-stability theory. However, if the fibre tension is negative, i.e. a compressive force, buckling of the fibre is to be expected (Tchavdarov, Yarin \& Radev 1993), so that we shall restrict attention to a positive fibre tension $\left(\sigma^{*}>0\right)$.

The structure of this paper is as follows. The Lagrangian model, for both axial-stretching and transverse-flow problems, is first described in $\S 2$ and the balance between fibre tension and surface tension needed to draw a fibre is discussed. Then, in $\S \S 3$ and 4 we present two carefully chosen case studies to show the mathematical framework in action. The case study of $\S 3$ involves a cross-section comprising an annular concatenation of sintering near-circular discs, and demonstrates the general applicability and power of our model formulation. The cross-section of the second case study in $\S 4$ is a concentric annulus. This second case study is presented in some detail since it affords us the opportunity to discuss ideas about the so-called 'forward' and 'inverse' problems of fibre drawing. Also, because it is a relatively simple solution which can be written down explicitly, the concentric annulus yields 
information that is difficult to obtain for fibres of other geometries. In addition, existing order-of-magnitude experimental data for drawing of circular tubes allows a rudimentary validation of our model; a more detailed experimental validation is in progress and will be presented elsewhere. A comparison of results for the two case studies turns out to be very instructive in relation to the questions of the uniqueness of solutions to the inverse problem and its inherent ill-posedness, and these matters are considered in $\S \S 5$ and 6. We conclude the paper with a more general discussion of how our results contribute to the challenging problem of solving the inverse problem for fibres having cross-sectional domains of higher connectivity.

\section{The mathematical model}

As shown by DeWynne et al. (1994) and Cummings \& Howell (1999), the slenderness of the fibre may be exploited to reduce the full three-dimensional Navier-Stokes model to coupled models for (i) the stretching flow along the axis of the fibre and (ii) the transverse flow in the cross-sectional plane. For ease of reading, we here give a summary of the derivation of these models.

Let the $x$-axis be directed along the axis of the fibre, in the direction of elongation, and let $y$ and $z$ be the coordinates in the cross-sectional plane. We denote the velocity vector and pressure by $\boldsymbol{u}=(u, v, w)$ and $p$, respectively, while $S(x)$ and $\Gamma(x)$ are the cross-sectional area and total perimeter length at position $x$. The boundary conditions are $u=U_{0}, S=S_{0}$ at $x=0$, and $u=U_{L}$ at $x=L$. The draw ratio is defined to be $D=U_{L} / U_{0}$ and, typically in fibre drawing, $D>1$. Table 1 shows typical parameter values for fibre drawing. The variables are scaled as follows, where asterisks denote the scaled quantities and $\epsilon=\sqrt{S_{0}} / L$ is assumed to be small (from table $1, \epsilon \leqslant 0.25$ ):

$$
\begin{gathered}
(x, y, z)=L\left(x^{*}, \epsilon y^{*}, \epsilon z^{*}\right), \quad t=\frac{L}{U_{0}} t^{*}, \\
(u, v, w)=U_{0}\left(u^{*}, \epsilon v^{*}, \epsilon w^{*}\right), \quad S=S_{0} S^{*}, \quad \Gamma=\epsilon L \Gamma^{*} .
\end{gathered}
$$

Like Griffiths \& Howell (2008), we allow that the fluid viscosity $\mu$ may vary with axial position:

$$
\mu=\mu_{0} \mu^{*}(x),
$$

where $\mu_{0}$ is a typical viscosity value. However, in any cross-section of the fibre the viscosity is assumed to be constant. Yarin et al. (1989), Griffiths \& Howell (2008) and, more recently, Taroni et al. (2013) considered a viscosity that depended on temperature and included a one-dimensional energy conservation equation to solve for the temperature as a function of axial position. Here we shall assume the viscosity to be a known function of axial position so that no energy-conservation model is required. Following the derivation of our model we will discuss this assumption and show that the final fibre geometry may be determined from the fibre tension, without knowledge of the temperature, so generalising the finding of Chen \& Birks (2013) for fibres containing isolated small holes. We also assume that the surface tension $\gamma$ is constant and define the scaled surface tension

$$
\gamma^{*}=\frac{\gamma}{\mu_{0} U_{0} \epsilon},
$$

which, from table 1 , is $O(1)$. Writing the unknown functions as power series of $\epsilon^{2}$, yields, at leading order, the two coupled models. 
Parameter

Surface tension

Viscosity

Density

Neck-down length

Feed speed

Draw ratio

Preform external radius

Preform cross-sectional area

\section{Symbol}

$$
\gamma
$$

$\mu_{0}$

$\rho$

$L$

$U_{0}$

D

$R_{P}$

$S_{0}$

$$
\text { Approx. value }
$$

Units

$$
0.2-0.3
$$$$
10^{5}-10^{6}
$$$$
\text { 2500-6000 }
$$

$$
0.04
$$$$
2.3 \times 10^{-5}
$$$$
10^{3}-10^{4}
$$

$5 \times 10^{-3}$

$5 \times 10^{-5}-8 \times 10^{-5}$
$\mathrm{N} \mathrm{m} \mathrm{m}^{-1}$

$\mathrm{Pa} \mathrm{s}$

$\mathrm{kg} \mathrm{\textrm {m } ^ { - 3 }}$

$\mathrm{m}$

$\mathrm{m} \mathrm{s}^{-1}$

m

$\mathrm{m}^{2}$

TABLE 1. Typical fibre-draw parameters.

\subsection{One-dimensional axial stretching model}

The axial flow is governed by one-dimensional mass and momentum conservation equations for $u(x)$ and $S(x)$ (Cummings \& Howell 1999; Wylie, Huang \& Miura 2007; Griffiths \& Howell 2008). Here we neglect gravity. For now we retain the inertial term but will see later that inertia too may be neglected because of the very small Reynolds number $R e=\rho U_{0} L / \mu_{0}=O\left(10^{-8}\right.$ ) (see table 1). Dropping asterisks on scaled variables, but retaining them on parameters for clarity of later discussion, the equations for conservation of mass and momentum are, respectively,

$$
\begin{gathered}
S_{t}+(S u)_{x}=0, \\
-\operatorname{Re} S\left(u_{t}+u u_{x}\right)+\left(3 \mu^{*} S u_{x}\right)_{x}+\frac{\gamma^{*}}{2} \Gamma_{x}=0 .
\end{gathered}
$$

At $x=0$ we have $S(0)=1$ and $u(0)=1$, while at $x=1$ we have $u(1)=D$. Because the flow in the Eulerian reference frame is steady, we can integrate (2.4) and (2.5), giving

$$
\begin{gathered}
u(x) S(x)=1, \\
-\operatorname{Re} u+3 \mu^{*} S u_{x}+\frac{\gamma^{*}}{2} \Gamma=6 \sigma^{*} .
\end{gathered}
$$

The constant value $6 \sigma^{*}$ is the axial tension in the fibre, scaled with $\mu_{0} U_{0} S_{0} / L$, i.e. $6 \sigma^{*}=\sigma L /\left(\mu_{0} U_{0} S_{0}\right)$; hereafter, we shall call $\sigma^{*}$ the (scaled) fibre tension parameter, or simply the fibre tension, as distinct from the surface tension. Mass conservation (2.6) immediately gives $S(1)=1 / D$.

Because we shall also use a Lagrangian reference frame in which the flow is unsteady, we also write the continuity equation (2.4) in the form

$$
\frac{\mathrm{D} S}{\mathrm{D} t}+S u_{x}=0
$$

where $\mathrm{D} S / \mathrm{D} t=\partial S / \partial t+u \partial S / \partial x$ is the material derivative (following a moving fluid particle) of $S$. This allows us to rewrite (2.7) as

$$
-\frac{R e}{S}-3 \mu^{*} \frac{\mathrm{D} S}{\mathrm{D} t}+\frac{\gamma^{*}}{2} \Gamma=6 \sigma^{*}
$$

where we have also substituted $u=1 / S$. The constant $\sigma^{*}$ is determined using the boundary condition $u(1)=D$ or $S(1)=1 / D$. For stretching of the fluid fibre so that $S(1)<1$, we require $D>1$. The typical range for $D$ is given in table 1 , from which we 
see that $\operatorname{Re} / S \leqslant \operatorname{Re} D=O\left(10^{-4}\right)$, which justifies our neglect of inertia from henceforth. Before we can write $\sigma^{*}$ in terms of $D$ we need to consider the two-dimensional model for the transverse flow, which determines the perimeter length $\Gamma(x)$.

\subsection{Two-dimensional transverse flow model}

The transverse flow in the cross-sectional plane is best found in terms of a Lagrangian axial coordinate $\tilde{x}$ and with the $y$ and $z$ coordinates in the cross-sectional plane scaled with $\sqrt{S}(\tilde{x}, \tilde{t})$ (Cummings \& Howell 1999):

$$
t=\tilde{t}, \quad x=\int_{0}^{\tilde{t}} u(\tilde{x}, T) \mathrm{d} T+\tilde{x}, \quad y=\sqrt{S} \tilde{y}, \quad z=\sqrt{S} \tilde{z}
$$

where we have assumed no rigid-body translation or rotation of each cross-section. We also transform the pressure and velocity thus:

$$
\begin{gathered}
p=-u_{x}(\tilde{x}, \tilde{t})+\frac{\gamma^{*}}{\sqrt{S(\tilde{x}, \tilde{t})}} \tilde{p}, \\
v=-\frac{1}{2} u_{x}(\tilde{x}, \tilde{t}) y+\frac{\gamma^{*} \tilde{v}}{\mu^{*}(\tilde{x}, \tilde{t})}, \quad w=-\frac{1}{2} u_{x}(\tilde{x}, \tilde{t}) z+\frac{\gamma^{*} \tilde{w}}{\mu^{*}(\tilde{x}, \tilde{t})},
\end{gathered}
$$

which corresponds to the scalings of Cummings \& Howell (1999) except that we allow a spatially varying viscosity and so include the viscosity in the scaling of the velocity components due to surface tension. The model for the transverse flow, in a reference frame that moves axially with a cross-section of the fibre, is then

$$
\begin{aligned}
\tilde{v}_{\tilde{y}}+\tilde{w}_{\tilde{z}} & =0, \\
\tilde{v}_{\tilde{y} \tilde{y}}+\tilde{v}_{\tilde{z} \tilde{z}} & =\tilde{p}_{\tilde{y}}, \\
\tilde{w}_{\tilde{y} \tilde{y}}+\tilde{w}_{\tilde{z} \tilde{z}} & =\tilde{p}_{\tilde{z}}, \\
\left(\mu^{*} \sqrt{S} / \gamma^{*}\right) G_{\tilde{t}}+\tilde{v} G_{\tilde{y}}+\tilde{w}_{\tilde{z}} & =0 \quad \text { on } G=0, \\
G_{\tilde{y}}\left(-\tilde{p}+2 \tilde{v}_{\tilde{y}}\right)+G_{\tilde{z}}\left(\tilde{v}_{\tilde{z}}+\tilde{w}_{\tilde{y}}\right) & =-\tilde{\kappa} G_{\tilde{y}} \quad \text { on } G=0, \\
G_{\tilde{y}}\left(\tilde{v}_{\tilde{z}}+\tilde{w}_{\tilde{y}}\right)+G_{\tilde{z}}\left(-\tilde{p}+2 \tilde{w}_{\tilde{z}}\right) & =-\tilde{\kappa} G_{\tilde{z}} \quad \text { on } G=0,
\end{aligned}
$$

where solutions of $G=0$ correspond to boundary points of the cross-section. By defining the reduced time $\tau$ (Cummings \& Howell 1999; Griffiths \& Howell 2008),

$$
\tau=\gamma^{*} \int_{0}^{\tilde{t}} \frac{\mathrm{d} \tilde{t}}{\mu^{*} \sqrt{S}}
$$

the kinematic boundary condition $(2.12 d)$ becomes

$$
G_{\tau}+\tilde{v} G_{\tilde{y}}+\tilde{w} G_{\tilde{z}}=0 \quad \text { on } G=0,
$$

and the transverse flow is given by the solution of a classical Stokes flow freeboundary problem on a domain with unit area and driven by the unit surface tension on the boundary. (It is common to refer to flows driven purely by the action of surface tension on their boundaries as viscous 'sintering' and we will sometimes use this terminology as shorthand for the physical mechanism active in the cross-plane.) 
To determine the change in $x(\tilde{x}, \tau)$ on a fixed cross-section as $\tau$ advances it is necessary to solve

$$
\frac{\partial x}{\partial \tau}=\frac{\partial x}{\partial \tilde{t}} \frac{\mathrm{d} \tilde{t}}{\mathrm{~d} \tau}=\frac{\mu^{*} u \sqrt{S}}{\gamma^{*}}=\frac{\mu^{*}}{\gamma^{*} \sqrt{S}} .
$$

One can solve the two-dimensional transverse-flow problem in reduced time $\tau$ using any method, analytical or numerical, that is appropriate for the particular geometry; for example see van de Vorst \& Mattheij (1995) and references therein. In this paper we focus on tubular fibres with a doubly connected cross-section. We study two cases in detail: where the cross-section is a concentric annulus and where the cross-section initially comprises $N \geqslant 3$ near-circular, just-touching discs of fluid placed symmetrically around an inner hole. These examples are chosen because the free boundary problem in the cross-plane in each case happens to admit solutions available in analytical form. The scaled perimeter length $\tilde{\Gamma}(\tau)$ can readily be calculated from such a solution thus allowing calculation of the perimeter length $\Gamma(\tau)=\sqrt{S} \tilde{\Gamma}(\tau)$ appearing in (2.9).

\subsection{Model coupling}

In coupling the one-dimensional axial flow and two-dimensional transverse flow models, Cummings \& Howell (1999) and Griffiths \& Howell (2008) work with the one-dimensional axial flow model expressed in terms of the Eulerian coordinate $x$. We, instead, write the one-dimensional axial flow model in terms of the reduced time variable $\tau$ which may then be solved for fibres of completely arbitrary geometry, including fibres with multiple air channels.

Without loss of generality we consider the fluid particle with Lagrangian coordinate $\tilde{x}=0$, i.e. that is at position $x=0$ at time $\tau=\tilde{t}=0$. Neglecting inertia and writing (2.9) in terms of the reduced time $\tau$ yields

$$
-\frac{3 \gamma^{*}}{\sqrt{S}} \frac{\mathrm{d} S}{\mathrm{~d} \tau}+\frac{\gamma^{*}}{2} \sqrt{S} \tilde{\Gamma}(\tau)=6 \sigma^{*},
$$

and putting $\chi(\tau)=\sqrt{S(\tau)}$ reduces (2.16) to a linear ordinary differential equation for $\chi$ :

$$
\frac{\mathrm{d} \chi}{\mathrm{d} \tau}-\frac{\chi}{12} \tilde{\Gamma}(\tau)=-\frac{\sigma^{*}}{\gamma^{*}} .
$$

Equation (2.17) is readily solved for $\tau>0$, subject to the initial condition $\chi(0)=1$, using the integrating factor

$$
H(\tau)=\exp \left(-\frac{1}{12} \int_{0}^{\tau} \tilde{\Gamma}\left(\tau^{\prime}\right) \mathrm{d} \tau^{\prime}\right) .
$$

This function $H(\tau)$ is a very important one that almost completely describes the stretching fibre. We find

$$
\chi(\tau)=\frac{1}{H(\tau)}\left(1-\frac{\sigma^{*}}{\gamma^{*}} \int_{0}^{\tau} H\left(\tau^{\prime}\right) \mathrm{d} \tau^{\prime}\right) .
$$

The relation between the reduced time $\tau$ and the physical position of the fluid particle $x$ is given by (2.15) and, for a known viscosity profile $\mu^{*}(x), 0 \leqslant x \leqslant 1$, it is convenient to use this in the form

$$
\frac{1}{\mu^{*}(x)} \frac{\partial x}{\partial \tau}=\frac{1}{\gamma^{*} \chi} .
$$


Then, defining

$$
m(x)=\int_{0}^{x} \frac{1}{\mu^{*}\left(x^{\prime}\right)} \mathrm{d} x^{\prime},
$$

we obtain

$$
\begin{aligned}
m(x) & =\frac{1}{\gamma^{*}} \int_{0}^{\tau} \frac{1}{\chi\left(\tau^{\prime}\right)} \mathrm{d} \tau^{\prime}=\frac{1}{\gamma^{*}} \int_{0}^{\tau} \frac{H\left(\tau^{\prime}\right)}{\left[1-\frac{\sigma^{*}}{\gamma^{*}} \int_{0}^{\tau^{\prime}} H(\xi) \mathrm{d} \xi\right]} \mathrm{d} \tau^{\prime} \\
& =-\frac{1}{\sigma^{*}} \log \left(1-\frac{\sigma^{*}}{\gamma^{*}} \int_{0}^{\tau} H\left(\tau^{\prime}\right) \mathrm{d} \tau^{\prime}\right) \\
& =-\frac{1}{\sigma^{*}} \log [H(\tau) \chi(\tau)] .
\end{aligned}
$$

Closed-form integration to evaluate $m(x)$ in (2.21) for a given viscosity profile $\mu^{*}(x)$ is sometimes possible: for the special case of constant viscosity $\mu^{*}(x)=1$ then $m(x)=x$ and we may write

$$
\exp \left(-\sigma^{*} x\right)=H(\tau) \chi(\tau)
$$

Finally, we define $\tau_{L}$ to be the total deformation time during which a cross-section travels from $x=0$ to $x=1$, so that $x\left(\tau_{L}\right)=1$. Then, from $(2.22 b)$ and using $\chi\left(\tau_{L}\right)=$ $1 / \sqrt{D}$

$$
\sigma^{*}=\frac{1}{M} \log \frac{\sqrt{D}}{H\left(\tau_{L}\right)},
$$

where $M=m(1)$ so that $1 / M$ is the harmonic mean of $\mu^{*}(x)$ over $0 \leqslant x \leqslant 1$, while (2.22a) yields

$$
\frac{H\left(\tau_{L}\right)}{\sqrt{D}}+\frac{\sigma^{*}}{\gamma^{*}} \int_{0}^{\tau_{L}} H(\tau) \mathrm{d} \tau=1 .
$$

The fibre-drawing problem involves essentially four parameters: $\tau_{L}, D, \sigma^{*}$ and $\gamma^{*}$. Because (2.24) and (2.25) provide two constraints, if two of these four parameters are specified, the model determines the remaining two. For example, if we wish to draw a particular fibre from a particular preform, the deformation time $\tau_{L}$ and draw ratio $D$ must take particular values; (2.24) and (2.25) then determine the (scaled) fibre tension $\sigma^{*}$ and surface tension $\gamma^{*}$ required to draw this fibre.

Since the surface tension scale is $\mu_{0} U_{0} \sqrt{S_{0}} / L$ (see (2.3)) and the fibre tension scale is $\mu_{0} U_{0} S_{0} / L$ (see (2.7)), the ratio of the physical fibre tension $\sigma$ and surface tension $\gamma$ is related to the ratio of the scaled quantities by

$$
\frac{\sigma}{\gamma}=6 \sqrt{S_{0}} \frac{\sigma^{*}}{\gamma^{*}} \text {. }
$$

Because the physical surface tension $\gamma$ is relatively insensitive to temperature and can be considered as a known quantity, the physical draw tension $\sigma$ can be obtained from (2.26) when $\sigma^{*} / \gamma^{*}$ is known. This means that, for drawing a particular fibre from a particular preform, (2.25) and (2.26) give the physical fibre tension $\sigma$, without needing to know the temperature of the glass or the length of the neck-down region. Conversely, if $\sigma$ is measured for a preform drawn at a given draw ratio $D$, then (2.25) and (2.26) give the final fibre geometry, also without any reference to the temperature or neck-down length. This was noted by Chen \& Birks (2013) for fibres with small, isolated holes. Here we see that it is true for any cross-sectional geometry. Since, in practical fibre drawing, the temperature in the glass and the neck-down length are 


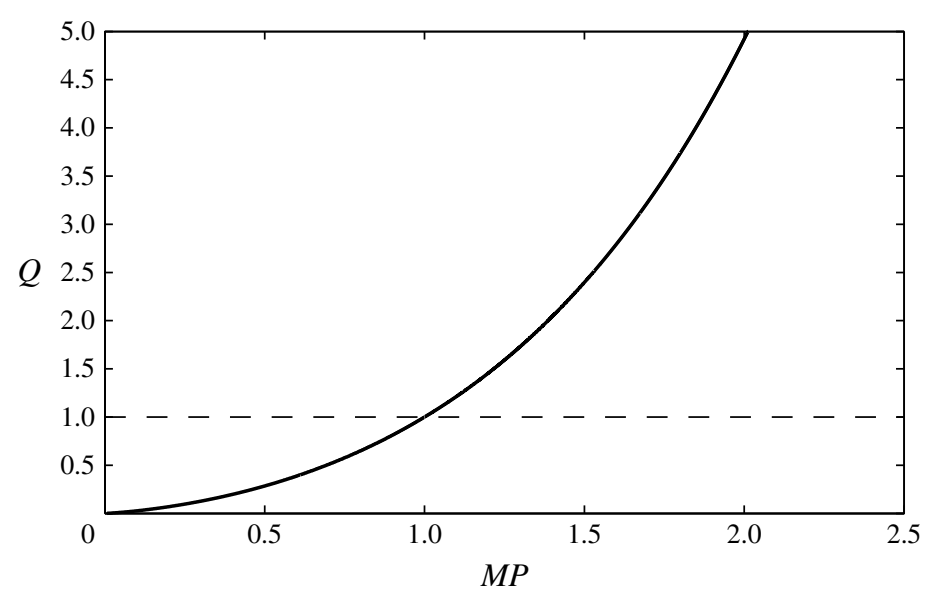

Figure 2. The relation between fibre tension measured by $Q$ and surface tension measured by $P$. The dashed curve shows the special solution $Q=1$ that satisfies (2.28) for any $P$. Here $M^{-1}$ is the harmonic mean of the viscosity over the neck-down length.

not known, while the fibre tension is usually measured, this is a crucial result. If the fibre geometry throughout the neck-down zone is of interest, then the problem would be much more difficult because the viscosity (or temperature) profile and the fibre geometry are interdependent, so that determination of the geometry requires solution of coupled flow and energy balance models; see, for example, Yarin et al. (1989), Griffiths \& Howell (2008) and Taroni et al. (2013). However, in fibre drawing it is the final geometry that is of interest so that we have the very important result that, for any preform geometry and known physical surface tension, the physical draw tension $\sigma$ required to produce a given allowable target geometry may be easily calculated from (2.25) and (2.26), without needing to know any other parameters.

Further insight into the relationship between the parameters may be obtained by defining

$$
P=\frac{\gamma^{*}}{\int_{0}^{\tau_{L}} H(\tau) \mathrm{d} \tau}, \quad Q=\frac{\sqrt{D}}{H\left(\tau_{L}\right)} .
$$

Equations (2.24) and (2.25) may then be written as a single equation,

$$
\frac{1}{Q}+\frac{1}{M P} \log Q=1 .
$$

Since $P$ measures the degree of sintering in the cross-plane due to surface tension effects while $Q$ measures the stretching of the fibre due to the fibre tension then (2.28) governs the balance between surface tension and axial tension needed to draw a particular fibre from a given preform. A graph of $Q$ against $M P$ is shown in figure 2 .

In the absence of surface tension so that $\gamma^{*}=0$ then $\tau_{L}=0$ implying that $H\left(\tau_{L}\right)=1$ and we have $Q=\sqrt{D}$ or $M \sigma^{*}=(\log D) / 2$, and deformation in the cross-section is due to stretching of the fibre alone.

It is interesting that $Q=1$ is a solution of (2.28) for any value of $M P$ and this corresponds to zero fibre tension $\sigma^{*}=0$ so that the draw ratio $D$ exactly counterbalances the axial effect of surface tension. For negligible surface tension $D=1$ and there is no deformation of the fibre geometry; otherwise surface tension 
is, effectively, responsible for all deformation, both sintering in the cross-plane and any stretching in the axial direction. Since $H\left(\tau_{L}\right) \leqslant 1$ (this follows from its definition (2.18)) then $Q=1$ can be a solution only for $D \leqslant 1$, a regime that is of little practical interest for fibre drawing, although it may be of interest for other applications of the model (for example, in preform fabrication). Note also that (2.27b) shows that, for non-negligible surface tension for which $H\left(\tau_{L}\right)<1$, we have $Q \geqslant 1$ for $H\left(\tau_{L}\right) \leqslant D \leqslant 1$. Thus a draw ratio smaller than unity does not imply a fibre in compression, a counter-intuitive result that we discuss again in $\S 4.3$ in the context of the annular fibre.

It is readily seen from figure 2 that $Q>1$ corresponds to $M P>1$ while $Q<1$ corresponds to $M P<1$. The latter implies $\sigma^{*}<0$, a negative fibre tension and, hence, axial compression of the fibre which is, in turn, expected to lead to buckling instability (Tchavdarov et al. 1993). Therefore, here we will insist on a positive fibre tension, $\sigma^{*}>0$ or $Q>1$. Also seen from figure 2 is the (exponential) increase in $Q$ with $M P$, for non-trivial solutions of (2.28). For some given $H(\tau)$ describing the surface-tensiondriven deformation in the cross-section, if $\tau_{L}$ is reduced while $\gamma^{*}$ is held constant (so that $M P$ increases) then this must be accompanied by an increase in the stretching rate, or $Q$, so that the new value of $\tau_{L}$ corresponds to $x=1$. Alternatively, holding $\tau_{L}$ constant and increasing the surface tension $\gamma^{*}$ implies a faster cross-plane evolution in physical time and, therefore, the need to increase the stretching rate in order to obtain the required cross-plane profile at $x=1$.

The formulation just described can be used for fibres of any cross-section, provided some method to solve the two-dimensional transverse-flow problem is available. For general cross-plane geometries numerical methods can be deployed. But, remarkably, it happens that the two-dimensional problem for the quasi-steady evolution of free-surface Stokes flows driven purely by surface tension is known to admit a rich variety of analytical solutions and we have chosen to focus on such solutions in this paper. Besides the geometrically simple case of a concentric annular tube there are known analytical solutions involving non-trivial geometries due to Hopper (1990), Richardson (1992), Crowdy \& Tanveer (1998a,b), Cummings, Howison \& King (1997), Richardson (2000), Crowdy (2003), among others. Without exception, these exact solutions derive from a complex-variable formulation of two-dimensional Stokes flow, one involving so-called Goursat functions common in plane elasticity (Langlois 1964; Muskhelishvili 1977).

The general formulation above can be combined with any of these analytical solutions; the latter can be used to compute the key governing function $H(\tau)$. To illustrate the power of our model formulation, we first consider in $\S 3$ a non-trivial geometry. Then, in $\S 4$ we look at the important case of a concentric annulus, which readily allows detailed analysis from which much may be learned.

\section{Case study 1: non-circular tubes}

As a first case study for the general framework we consider non-circular tubes formed by the sintering and drawing of $N \geqslant 3$ near-circular discs of fluid placed in a rotationally symmetric array around an inner hole and just touching (figure $3 a$ top). Our principal motivation for choosing this example is the fact that we can make use of known exact solutions for the evolution of such doubly connected cross-plane geometries (Crowdy \& Tanveer 1998b; Richardson 2000; Crowdy 2003) to determine $H(\tau)$. While this example is a good showcase for the general framework described above it is also not without physical interest: it arises in the making of a fibre coupler, a process whereby multiple fibres are heated and pulled in order to merge them into a single fibre (Howell 1994; Cummings \& Howell 1999). 
(a)

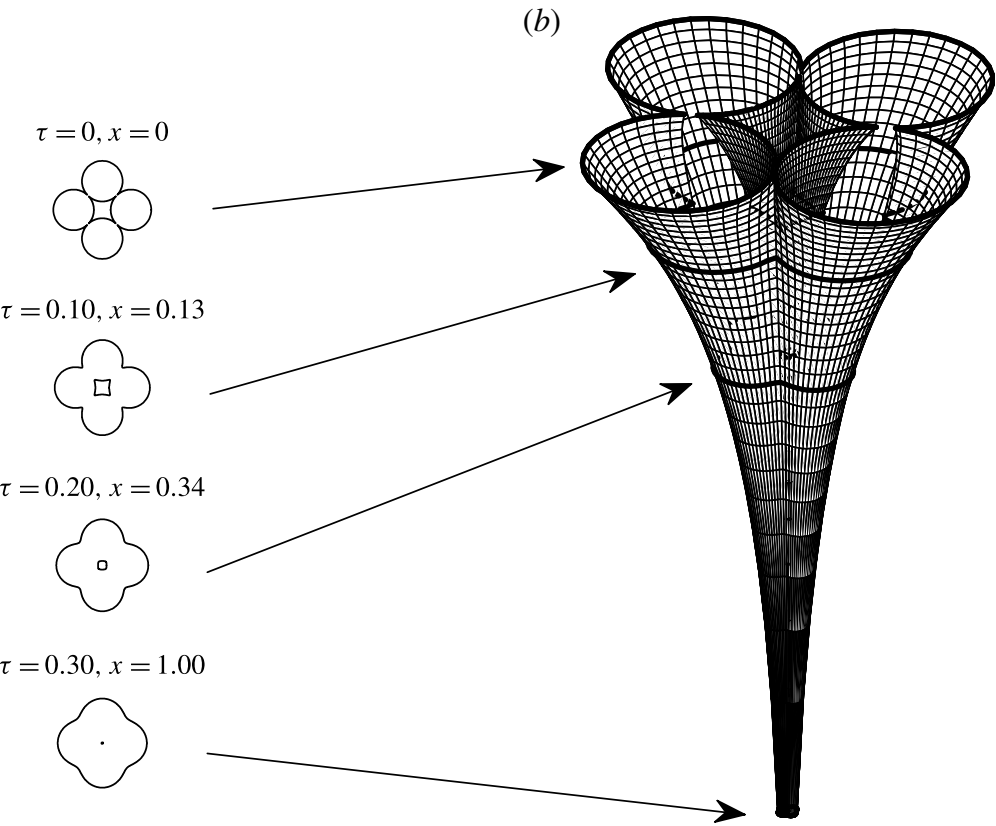

FIGURE 3. (a) Snapshots, at various values of $\tau$ and their associated $x(\tau)$ values, of the evolution of the cross-plane solution described in $\S 3.1$; the curves show the images of $|\zeta|=1$ and $|\zeta|=\rho(\tau)$ under the map (3.1a). (b) A visualisation of the three-dimensional fibre. In this figure, $D=400, \gamma^{*}=0.9$, and $\sigma^{*}=3.1$. The transverse dimensions have been stretched relative to the axial dimension; constant viscosity is assumed.

\subsection{The transverse flow}

Initially the two-dimensional cross-section is a doubly connected domain. A convenient form of the explicit solution for the evolution of the doubly connected cross-section, before hole closure, has been given by Crowdy (2003). At a critical time (dependent on $N$, the values of which are recorded by Crowdy 2003) the central hole closes up, rendering the domain simply connected, although, since we are principally concerned here with annular fibres, we terminate our calculations before this hole closure time. The shape of the cross-plane is given as the image of a pre-image annulus $\rho(\tau)<|\zeta|<1$ in a parametric complex $\zeta$-plane under the time-evolving conformal map

$$
z(\zeta, \tau)=A(\tau) f(\zeta, \tau), \quad f(\zeta, \tau)=\zeta \frac{P_{N}\left(\zeta \rho(\tau)^{2 / N} a(\tau)^{-1}, \rho(\tau)\right)}{P_{N}\left(\zeta a(\tau)^{-1}, \rho(\tau)\right)},
$$

where we can choose any $N \geqslant 3$ (corresponding to the number of near-circular cylinders in the annular array). Here,

$$
P_{N}(\zeta, \rho) \equiv\left(1-\zeta^{N}\right) \prod_{k=1}^{\infty}\left(1-\rho^{2 k N} \zeta^{N}\right)\left(1-\rho^{2 k N} \zeta^{-N}\right), \quad \hat{P}_{N}(\zeta, \rho) \equiv P_{N}(\zeta, \rho) /\left(1-\zeta^{N}\right)
$$

with

$$
A(\tau)=\sqrt{\frac{\hat{P}_{N}(1, \rho(\tau))}{\pi P_{N}\left(\rho(\tau)^{2 / N}, \rho(\tau)\right) f^{\prime}\left(a(\tau)^{-1}, \tau\right)}},
$$


where a prime denotes the partial derivative with respect to $\zeta$, that is, $f^{\prime}(\zeta, \tau)=$ $\partial f(\zeta, \tau) / \partial \zeta$. Condition (3.1c) ensures that the fluid cross-section always has unit area as required by the scaling of the transverse-flow problem (2.10). The above equations are reproductions of (2.16), (2.18) and (3.2) of Crowdy (2003). The time-evolving parameters $\rho(\tau)$ and $a(\tau)$ satisfy the ordinary differential equations (Crowdy 2003):

$$
\begin{gathered}
\frac{\mathrm{d} \rho}{\mathrm{d} \tau}=-\frac{\rho}{4 \pi \mathrm{i}}\left(\oint_{|\eta|=1} \frac{\mathrm{d} \eta}{\eta} \frac{1}{\left|z^{\prime}(\eta, \tau)\right|}+\oint_{|\eta|=\rho} \frac{\mathrm{d} \eta}{\eta} \frac{1}{\rho\left|z^{\prime}(\eta, \tau)\right|}\right), \\
\frac{\mathrm{d} a}{\mathrm{~d} \tau}=a I\left(a^{-1}, \tau\right),
\end{gathered}
$$

where, for brevity, we have suppressed the dependence of these parameters on $\tau$ in our notation. The definition of the function $I(\zeta, \tau)$ is given in appendix A.

For each $N$ the initial values of the parameters $\rho, a$ and $A$ corresponding to $N$ near-circular cylinders that are just touching are recorded in table 1 of Crowdy (2003). The ordinary differential equations $(3.2 a)$ and $(3.2 b)$ are solved numerically using the Runge-Kutta fourth-order method, with $A(\tau)$ obtained from the algebraic condition (3.1c). All integrals around the circles $|\eta|=1$ and $|\eta|=\rho$ are evaluated using the trapezoidal rule with 1024 equally spaced points. The product in $(3.1 b)$ is truncated after 100 factors.

It should be emphasised that, in contrast to the analytic solution of Griffiths \& Howell $(2007,2008)$ which is an asymptotic solution approximating the evolution of viscida with sufficiently thin walls, the solution for $N$ sintering cylinders just described above is exact and no approximation is involved in solving for the cross-plane evolution.

\subsection{Axial stretching and the three-dimensional neck-down geometry}

To couple the transverse flow to the axial flow we must determine the total perimeter length $\tilde{\Gamma}(\tau)$ from the transverse-flow solution. For this example we can make use of the formulae

$$
\tilde{\Gamma}(\tau)=\oint \mathrm{d} s=\oint|\mathrm{d} z|=\oint\left|z^{\prime}(\zeta, \tau)\right| \mathrm{d} \zeta \mid=-\mathrm{i} \oint_{|\zeta|=1} \frac{\left|z^{\prime}(\zeta, \tau)\right|}{\zeta} \mathrm{d} \zeta-\mathrm{i} \rho \oint_{|\zeta|=\rho} \frac{\left|z^{\prime}(\zeta, \tau)\right|}{\zeta} \mathrm{d} \zeta
$$

which only requires knowledge of $z^{\prime}(\zeta, \tau)$ which can, in turn, be computed from knowledge of the conformal map (3.1a); details on this calculation are given in appendix A. Knowledge of $\tilde{\Gamma}(\tau)$ facilitates calculation of the function $H(\tau)$ as defined in (2.18). The latter function is all that is needed from the transverse-flow problem to determine the three-dimensional fibre geometry.

We choose the value of $\tau_{L}$ (which effectively dictates what cross-sectional profile we want at the end of the draw) and set the draw ratio $D$, thus setting the value of $Q$ from (2.27b). Parameter $P$ is then found by solving (2.28), allowing $\gamma^{*}$ to be calculated from $(2.27 a)$. Finally $\sigma^{*}$ can be obtained from (2.24).

With the function $H(\tau)$ and the parameters $\gamma^{*}$ and $\sigma^{*}$ known, $S(\tau)$ can be calculated from (2.19) and $m(x(\tau))$ from (2.22b). For a given choice of viscosity $\mu^{*}(x)$, this gives $x(\tau)$ and $S(x)$, allowing the full three-dimensional fibre geometry to be obtained.

The evolution of an example cross-section for $N=4$, and with draw ratio $D=400$ and viscosity $\mu^{*}=1$, is shown in figure 3; the cross-sectional profiles, labelled with their respective values of $\tau$, and the fully three-dimensional realisation of the simulation are juxtaposed. 


\section{Case study 2: circular tubes}

As a second case study we apply the method of $\S 2$ to drawing of circular tubes for which the cross-sectional domain is the region between two concentric circles. This problem was considered by Fitt et al. (2002) using a one-dimensional Navier-Stokes model but without coupling to a model for the transverse flow, so that a complete solution was not determined, while Yarin et al. (1994) obtained numerical solutions for thin-walled tubes in the case of non-negligible surface tension. A thin-walled circular tube is one of the cases for which the approach of Griffiths \& Howell (2008) is appropriate and, indeed, those authors showed how to retrieve the results of Fitt et al. (2002) within their own formulation. However, the approach described in the current paper yields a more complete solution without any restrictions on the possible wall thicknesses. Furthermore, this relatively simple case admits a closed-form solution and analysis of it will inform future investigations of more complex geometries requiring numerical solution. In particular, from a study of this annular tube example in explicit detail, it is possible to gain some important insights into the solution of the so-called 'forward' and 'inverse' problems. The first case study demonstrated how to determine a full three-dimensional fibre from knowledge of a given initial preform cross-section, which is the 'forward problem'. In many practical applications, however, it is very often the final fibre geometry that is known and it is desirable to ascertain the appropriate initial preform geometry, as well as the associated experimental draw parameters, needed to produce it. This 'inverse problem' is, typically, much more difficult to solve than the forward problem, but it is also more important in practice.

Finally, this simple example affords us a preliminary validation of our model against experimental data and this is described in $\$ 4.4$.

\subsection{The transverse flow}

We first obtain the transverse flow by solving for the surface-tension-driven flow in an annular domain of unit area, i.e. $S=1$. For this it is convenient to use polar spatial coordinates $(r, \theta)$, where $r$ is the radial coordinate and $\theta$ is the azimuthal coordinate.

Let $\tilde{r}=R(\tau)$ be the radius of the outer circular boundary and $\tilde{r}=\rho(\tau) R(\tau)$ be the radius of the inner circular boundary. Then

$$
1=\pi R^{2}\left(1-\rho^{2}\right) \Rightarrow R=\frac{1}{\sqrt{\pi\left(1-\rho^{2}\right)}} .
$$

The initial geometry is given by $\rho_{0}=\rho(0)$ which determines $R_{0}=R(0)$ or vice versa. Alternatively we may specify the final geometry $\rho_{L}=\rho\left(\tau_{L}\right)$ or $R_{L}=R\left(\tau_{L}\right)$.

In polar coordinates, the axisymmetric forms of $(2.12 a)-(2.12 c),(2.12 e)-(2.12 c)$, to be solved for the radial velocity $\tilde{v}(\tilde{r})$ and the pressure $\tilde{p}(\tilde{r})$, are

$$
\begin{gathered}
\frac{1}{\tilde{r}} \frac{\partial}{\partial \tilde{r}}(\tilde{r} \tilde{v})=0, \\
\frac{\partial \tilde{p}}{\partial \tilde{r}}+\frac{1}{\tilde{r}} \frac{\partial}{\partial \tilde{r}}\left(\tilde{r} \frac{\partial \tilde{v}}{\partial \tilde{r}}\right)-\frac{\tilde{v}}{\tilde{r}^{2}}=0, \\
-\tilde{p}+2 \frac{\partial \tilde{v}}{\partial \tilde{r}}=-\tilde{\kappa} \quad \text { on } G=0, \\
\frac{\partial G}{\partial \tilde{\tau}}+\tilde{v}=0 \quad \text { on } G=0,
\end{gathered}
$$


where $G=G_{O}+G_{I}, G_{O}=\tilde{r}-R(\tau)$ and $G_{I}=\rho(\tau) R(\tau)-\tilde{r}$, and $\tilde{\kappa}=1 / R$ on $G_{O}=0$ and $\tilde{\kappa}=-1 /(\rho R)$ on $G_{I}=0$. Solution of $(4.2 a)-(4.2 c)$ yields

$$
\tilde{v}=-\frac{\rho R}{2 \tilde{r}(1-\rho)}, \quad \tilde{p}=\frac{1}{R(1-\rho)},
$$

while the kinematic condition $(4.2 d)$ gives

$$
\frac{\mathrm{d} R}{\mathrm{~d} \tau}=-\frac{\rho}{2(1-\rho)}, \quad \frac{\mathrm{d}}{\mathrm{d} \tau}(\rho R)=-\frac{1}{2(1-\rho)}
$$

and, on summing and integrating,

$$
R(1-\rho)=\frac{\tau}{2}+\alpha_{0}
$$

for some constant $\alpha_{0}$ which quantifies the initial geometry. Substituting for $R$ from (4.1) gives, with a little manipulation,

$$
\alpha(\tau)=\sqrt{\frac{1-\rho}{\pi(1+\rho)}}=\alpha_{0}\left(\frac{\tau}{2 \alpha_{0}}+1\right),
$$

where $\alpha_{0}=\alpha(0)$, from which we find

$$
\rho(\tau)=\frac{1-\pi \alpha^{2}}{1+\pi \alpha^{2}}
$$

and

$$
\tilde{\Gamma}(\tau)=2 \pi R(1+\rho)=\frac{2}{\alpha}=\frac{2}{\alpha_{0}}\left(\frac{\tau}{2 \alpha_{0}}+1\right)^{-1} .
$$

The inner hole closes when $\rho=0(\alpha=1 / \sqrt{\pi})$ at $\tau=\tau_{\max }$, where

$$
\tau_{\max }=2\left(\frac{1}{\sqrt{\pi}}-\alpha_{0}\right),
$$

and beyond this time this model is not valid ( $\rho(\tau)$ becomes negative) and must be replaced by a unit circle with no interior holes. Since the aim is to preserve interior holes, we do not consider $\tau \geqslant \tau_{\max }$.

Putting $\alpha\left(\tau_{L}\right)=\alpha_{L}$ into (4.6) gives

$$
\tau_{L}=2\left(\alpha_{L}-\alpha_{0}\right) .
$$

For given $\alpha_{L}<1 / \sqrt{\pi}\left(\rho_{L}>0\right)$, we need not be concerned with closure of the interior hole. However, we now require $\alpha_{0}>0\left(\rho_{0}<1\right)$ and, therefore, $\tau_{L}<2 \alpha_{L}$. Note that $\alpha_{L}>\alpha_{0}$, i.e. $\alpha_{L} / \alpha_{0}>1$.

\subsection{Axial stretching and the three-dimensional neck-down geometry}

We next determine the change in cross-sectional area with axial position $x$ due to both surface tension and axial stretching, for some given viscosity profile $\mu^{*}(x)$ and, hence, function $m(x)$ and value $M=m(1)$, where $M^{-1}$ is the harmonic mean of $\mu^{*}(x)$ over the region $[0,1]$.

We start with the forward problem and therefore take $\alpha_{0}$ to be known. From (2.18) and (4.8), we have

$$
H(\tau)=\exp \left(-\frac{1}{12} \int_{0}^{\tau} \tilde{\Gamma}\left(\tau^{\prime}\right) \mathrm{d} \tau^{\prime}\right)=\left(\frac{\tau}{2 \alpha_{0}}+1\right)^{-1 / 3}
$$


It is then easy to establish that

$$
\begin{gathered}
\int_{0}^{\tau} H\left(\tau^{\prime}\right) \mathrm{d} \tau^{\prime}=3 \alpha_{0}\left[\left(\frac{\tau}{2 \alpha_{0}}+1\right)^{2 / 3}-1\right], \\
S(\tau)=\left(\frac{\tau}{2 \alpha_{0}}+1\right)^{2 / 3}\left\{1-\frac{3 \alpha_{0} \sigma^{*}}{\gamma^{*}}\left[\left(\frac{\tau}{2 \alpha_{0}}+1\right)^{2 / 3}-1\right]\right\}^{2}, \\
m(x(\tau))=-\frac{1}{\sigma^{*}} \log \left\{1-\frac{3 \alpha_{0} \sigma^{*}}{\gamma^{*}}\left[\left(\frac{\tau}{2 \alpha_{0}}+1\right)^{2 / 3}-1\right]\right\},
\end{gathered}
$$

with expressions for $u(\tau)$ and $x(\tau)$ then following directly from (1.2).

Given two of the four parameters $\tau_{L}, D, \sigma^{*}, \gamma^{*}$, we can compute the remaining two parameters using the relations

$$
\begin{gathered}
Q=\sqrt{D}\left(\frac{\tau_{L}}{2 \alpha_{0}}+1\right)^{1 / 3}=\sqrt{D}\left(\frac{\alpha_{L}}{\alpha_{0}}\right)^{1 / 3}, \\
P=\frac{\gamma^{*}}{3 \alpha_{0}}\left[\left(\frac{\tau_{L}}{2 \alpha_{0}}+1\right)^{2 / 3}-1\right]^{-1}=\frac{\gamma^{*}}{3 \alpha_{0}}\left[\left(\frac{\alpha_{L}}{\alpha_{0}}\right)^{2 / 3}-1\right]^{-1},
\end{gathered}
$$

together with the relation (2.28) between $P$ and $Q$. The final fibre geometry $\alpha_{L}$ follows from (4.10).

Solutions for any given value of $\alpha_{0}$ may be computed over a grid of points $\left(\tau_{L}, D\right)$, $0 \leqslant \tau_{L} \leqslant \tau_{\max }, 0 \leqslant D \leqslant D_{\max }$, for some chosen value $D_{\max }$ and $\tau_{\max }$ given by (4.9). For a given final geometry, hence choice of $D$ and $\tau_{L}$, there is a unique set of parameters $\gamma^{*}$ and $\sigma^{*}$.

The inverse problem is solved similarly. We take $\alpha_{L}$ to be known and compute solutions over a grid of points $\left(\tau_{L}, D\right), 0 \leqslant \tau_{L} \leqslant 2 \alpha_{L}, 0 \leqslant D \leqslant D_{\max }$, for some chosen value $D_{\max }$.

Figures 4 and 5 show solutions of the forward problem for $\alpha_{0}=1 / \sqrt{3 \pi}\left(\rho_{0}=0.5\right)$. Figure $4(a)$ shows the geometry over $0 \leqslant x \leqslant 1$ for drawing of a fibre with parameters $\gamma^{*}=0.5$ and $\sigma^{*}=2$, and with a viscosity $\mu^{*}=1$ that is independent of spatial position $x$ so that $m(x)=x$ and $M=1$. To illustrate a non-constant viscosity, figure $4(b)$ shows the physical geometry for the same parameters and with the viscosity profile $\mu^{*}(x)=\exp (x-0.5)$ so that $m(x)=\exp (0.5)[1-\exp (-x)]$ and $M=2 \sinh (0.5)$. Now we suppose that the scaled surface tension $\gamma^{*}$ is fixed by the properties of the fibre material and the setup of the drawing tower and, in figure 5(a), show contours of $M \gamma^{*}$ in $\left(D, \tau_{L}\right)$ space for $0<D \leqslant 100$. Figure $5(b)$ shows the corresponding $M \sigma^{*}$-contours. By plotting contours of viscosity-modified surface tension and fibre tension, $M \gamma^{*}$ and $M \sigma^{*}$, rather than just $\gamma^{*}$ and $\sigma^{*}$, we obtain plots that are applicable for any viscosity profile. For given values of the draw ratio $D=1 / S_{L}$ and $M \gamma^{*}$ we may read $\tau_{L}$ from figure 5(a) and, hence, compute the final geometry $\alpha_{L}$. Figure $5(b)$ then gives the fibre tension $M \sigma^{*}$ needed to draw this fibre from a preform with geometry $\alpha_{0}$.

Next we choose $\alpha_{L}=\sqrt{2 /(3 \pi)}\left(\rho_{L}=0.2\right)$ and solve the inverse problem to determine the initial conditions and draw parameters that will yield this fibre geometry. Figure 6(a) shows contours of $M \gamma^{*}$ in $\left(D, \tau_{L}\right)$ space. We see that for a given value of $\gamma^{*}$, a given fibre geometry, specified by $\alpha_{L}$ and $D$, may, in general, be drawn from two different initial geometries. Because the contours are, in fact, parameterised by the 

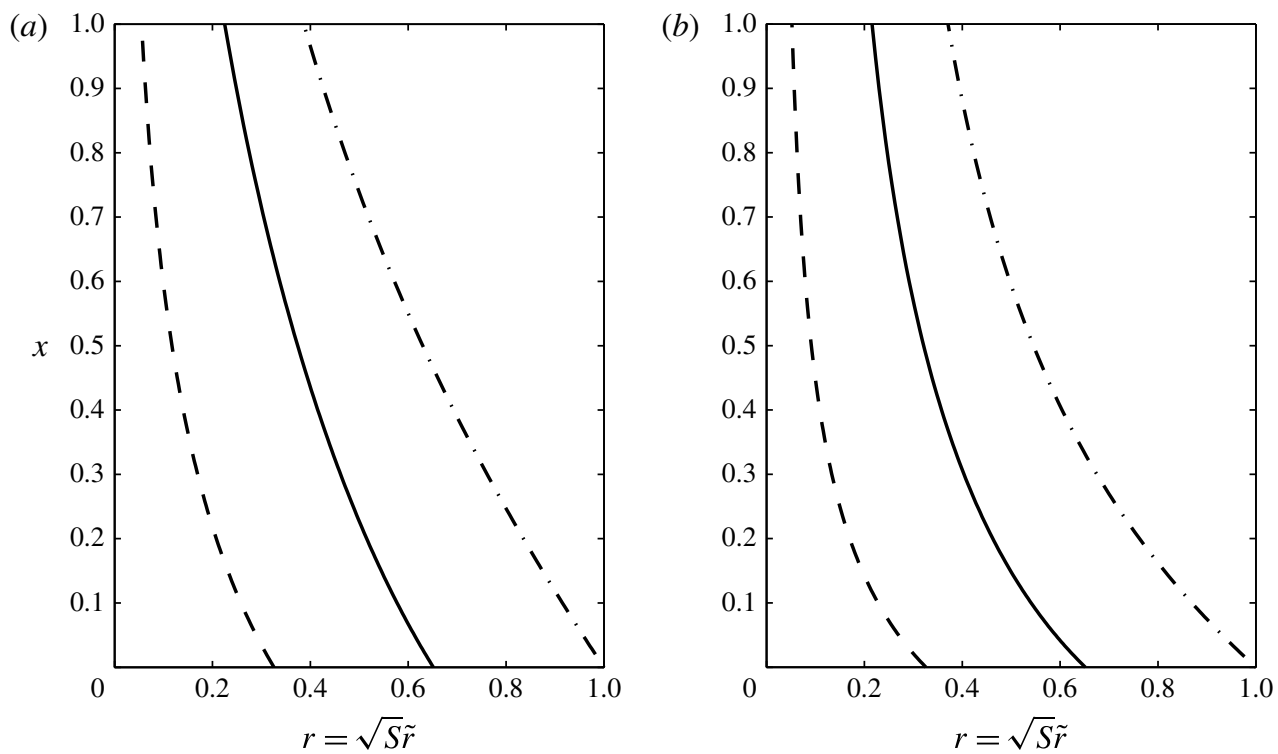

FIgURE 4. Geometry over $0 \leqslant x \leqslant 1$ for $\alpha_{0}=1 / \sqrt{3 \pi}\left(\rho_{0}=0.5\right), \gamma^{*}=0.5$ and $\sigma^{*}=2$, with viscosity profiles $(a) \mu^{*}(x)=1$ and $(b) \mu^{*}(x)=\exp (x-0.5)$. The solid curve is the outer boundary $r(x)=R \sqrt{S}$, the dashed curve is the inner boundary $r(x)=\rho R \sqrt{S}$, and the dash-dot curve shows the cross-sectional area $S(x)$.
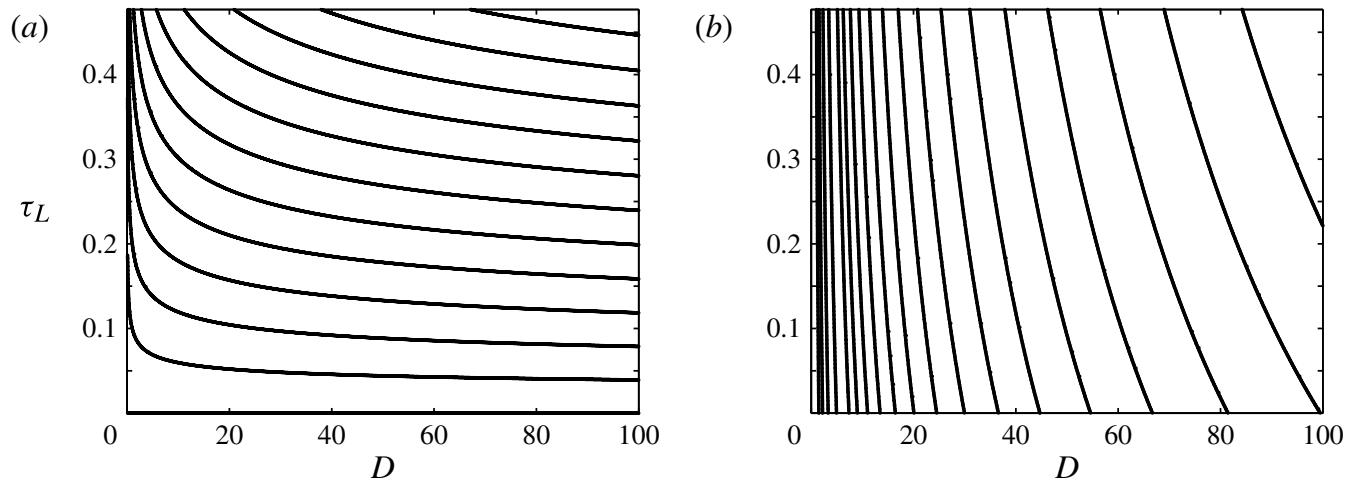

FIGURE 5. Solutions of the forward problem for $\alpha_{0}=1 / \sqrt{3 \pi}\left(\rho_{0}=0.5\right)$ shown as contours of $M \gamma^{*}\left(D, \tau_{L}\right)$ and $M \sigma^{*}\left(D, \tau_{L}\right):(a) M \gamma^{*}=0.1,0.2, \ldots, 1.1$ (bottom to top) and (b) $M \sigma^{*}=$ $0.2,0.4, \ldots, 1,1.1,1.2, \ldots, 2.4$ (left to right). For a given final geometry, i.e. choice of $D$ and $\tau_{L}$, and a given harmonic-mean viscosity $M^{-1}$, there is a unique set of parameters $\gamma^{*}, \sigma^{*}$.

fibre tension $\sigma^{*}$, the two initial geometries correspond to using different values of the fibre tension in the draw process. Figure $6(b)$ shows contours of $M \sigma^{*}$. The change in $M \sigma^{*}$ as we traverse an $M \gamma^{*}$-contour may be seen by superimposing these two plots. For any fibre geometry $\alpha_{L}$, figures similar to figure $6(a, b)$ can, therefore, be used to determine all fibre-draw parameters and the initial geometry. Another representation of the fibre drawing information is to plot contours of $M \gamma^{*}$ in $\left(D, M \sigma^{*}\right)$ space, as done 

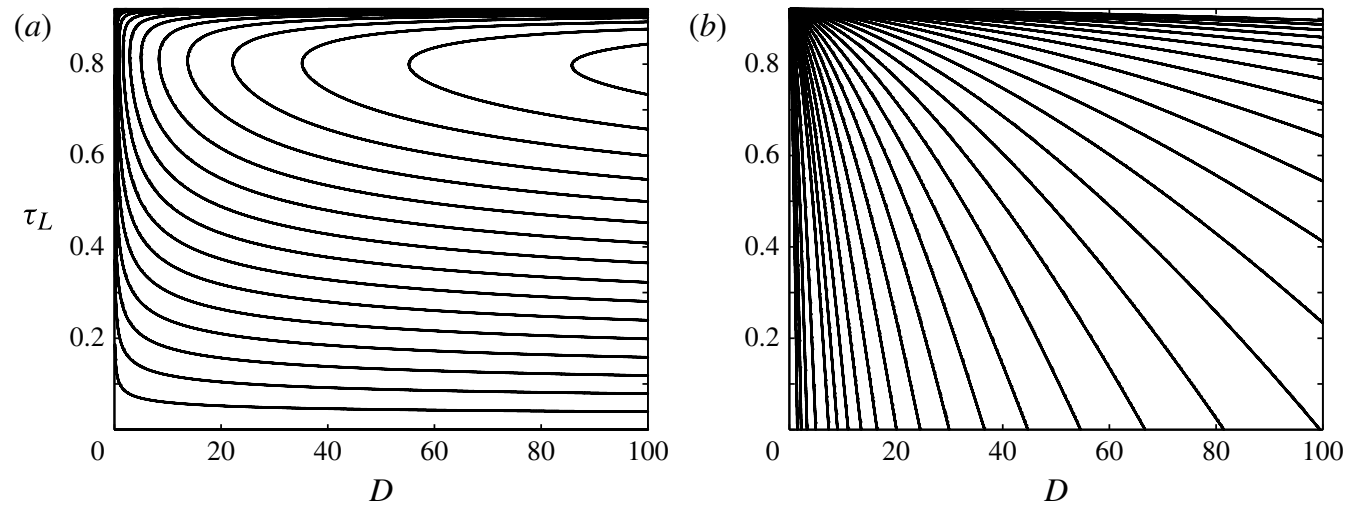

FIgURE 6. Solutions of the inverse problem for $\alpha_{L}=\sqrt{2 /(3 \pi)}\left(\rho_{L}=0.2\right)$ shown as contours of $M \gamma^{*}\left(D, \tau_{L}\right)$ and $M \sigma^{*}\left(D, \tau_{L}\right):(a) M \gamma^{*}=0.1,0.2, \ldots, 1.6$ (bottom to top) and $(b) M \sigma^{*}=0.2,0.4, \ldots, 1,1.1,1.2, \ldots, 3.5$ (left to right). For a given final geometry, i.e. choice of $D$, and a given harmonic-mean viscosity $M^{-1}$, there are many choices of initial condition $\left(\tau_{L}\right)$ and parameters $\gamma^{*}, \sigma^{*}$ that will yield the desired fibre. For a given value of $\gamma^{*}$, there are, in general, two choices of initial condition and fibre tension $\sigma^{*}$ that will yield the desired fibre.

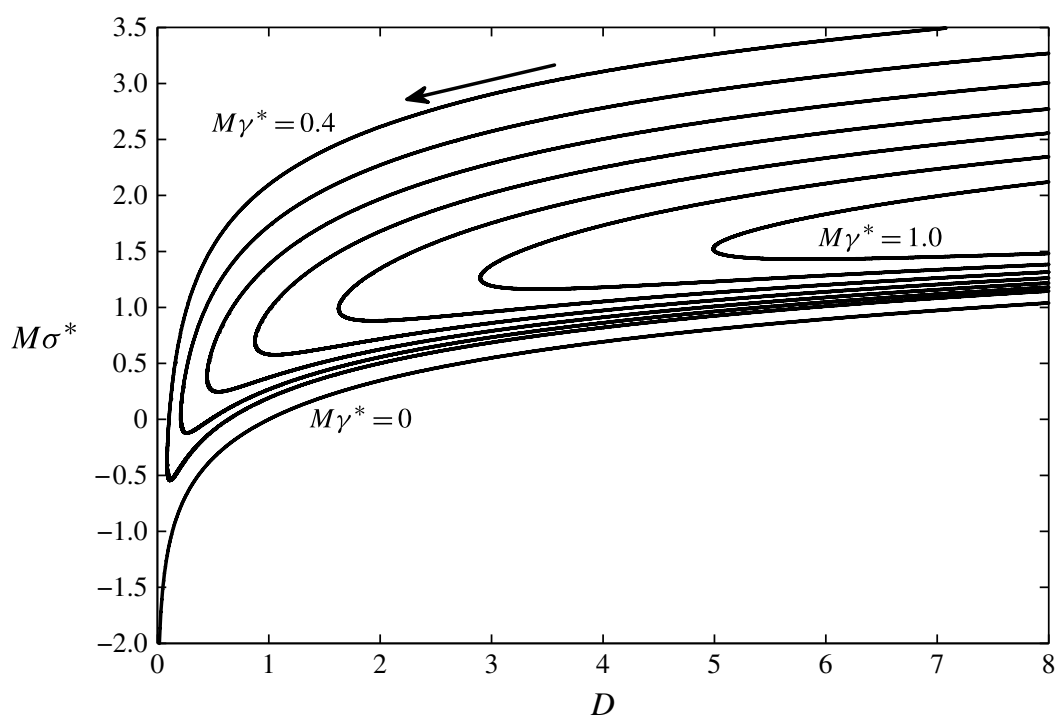

FIgURE 7. Viscosity-modified fibre tension $M \sigma^{*}$ versus draw ratio $D$ for $\alpha_{L}=\sqrt{2 /(3 \pi)}$ $\left(\rho_{L}=0.2\right)$ and $M \gamma^{*}=0,0.4,0.5, \ldots, 1.0$. The curves are parameterised by $\tau_{L}$ (equivalently $\alpha_{0}$ ) and the arrow shows the direction of decreasing $\tau_{L}$ (increasing $\alpha_{0}$ ).

in Griffiths \& Howell (2008) for drawing of thin-walled tubes. Figure 7 shows this for drawing of annular fibres with $\alpha_{L}=\sqrt{2 /(3 \pi)}$, i.e. a tube which does not have a thin wall. These curves are parameterised by the deformation time $\tau_{L}$ (equivalently $\alpha_{0}$ ).

Having solved a forward or inverse fibre-drawing problem we may compute $\rho(\tau)$ and $R(\tau), 0 \leqslant \tau \leqslant \tau_{L}$. Physical external and internal radii are obtained by multiplying by $\sqrt{S_{0} S(\tau)}$, where $S_{0}$ is the physical area at $x=0$, and $S(\tau)$ is the cross-sectional area 
scaled by $S_{0}$ which is given by $(4.11 c)$. We note that $\rho(\tau)$ and, hence, $R(\tau)$ are strictly monotonic decreasing functions of $\tau$. However, $S$ may not be a decreasing function of $\tau$. This suggests the question: under what conditions will the physical fibre radius be smaller than the physical preform radius? For this we require

$$
R_{0}>\sqrt{S_{L}} R_{L} \Rightarrow \sqrt{D}>\frac{R_{L}}{R_{0}},
$$

or

$$
\sqrt{D}>f\left(\alpha_{0}, \alpha_{L}\right), \quad f\left(\alpha_{0}, \alpha_{L}\right)=\frac{\alpha_{0}\left(1+\pi \alpha_{L}^{2}\right)}{\alpha_{L}\left(1+\pi \alpha_{0}^{2}\right)} .
$$

Now, $0<\alpha_{0}<1 / \sqrt{\pi}$ and $\alpha_{0} \leqslant \alpha_{L}<1 / \sqrt{\pi}$ and, for given $\alpha_{0}, f\left(\alpha_{0}, \alpha_{L}\right)$ is a decreasing function of $\alpha_{L}$ over the physically meaningful range of $\alpha_{L}$, taking its maximum value $f=1$ when $\alpha_{L}=\alpha_{0}$. Then, for $f\left(\alpha_{0}, \alpha_{L}\right)<\sqrt{D}<1$ the final 'fibre' will have smaller physical external radius than the preform, while the cross-sectional area will be larger than that of the initial preform. For $\sqrt{D}>1$ both the physical external radius and the cross-sectional area of the final fibre will be smaller than those of the preform, and this is the case for fibre drawing for which $D \gg 1$.

\subsection{Fibre buckling and other stability issues}

As discussed in the Introduction, we are not concerned with an upper limit on the draw ratio to avoid draw resonance, because of the large draw ratios used successfully in practice. However, as also noted earlier, buckling is to be expected if the fibre tension is negative, i.e. a compressive force (Tchavdarov et al. 1993), so that we do require $\sigma^{*}>0$ or $Q>1$ or $\sqrt{D}>\left(\alpha_{0} / \alpha_{L}\right)^{1 / 3}$. Since $\alpha_{0} / \alpha_{L} \leqslant 1$, this is certainly true for $D>1$ and, hence, in fibre drawing. Although not relevant to practical fibre drawing, it is interesting to note from (4.14) that $f\left(\alpha_{0}, \alpha_{L}\right)>\left(\alpha_{0} / \alpha_{L}\right)^{1 / 3}$, so that for draw ratios in the range $f\left(\alpha_{0}, \alpha_{L}\right)<\sqrt{D}<1$, just discussed above, for which the 'fibre' has smaller external radius and larger cross-sectional area than the preform, the fibre tension is positive and buckling instability is not of concern. This is a result of surface tension; when the effect of surface tension is small so that $\alpha_{L} \approx \alpha_{0}$ we essentially have compression of the fibre for $D<1$.

As seen in figure 7 and as noted by Griffiths \& Howell (2008), for any given value of $\gamma^{*}$ (taking the harmonic-mean viscosity $M^{-1}$ to be a known constant) there is a region in the vicinity of the minimum value of the draw ratio $D$ in which the fibre tension $\sigma^{*}$ is a decreasing function of $D$. Griffiths \& Howell (2008) reason that such a region 'surely cannot be stable' and also claim that the upper branch of the fibre-tension versus draw-ratio curve for a given value of scaled surface tension $\gamma^{*}$ is likely to represent unstable solutions because of 'physically implausible behaviour'. These conclusions seem to be motivated by an assumption that the modelling should yield a unique stable initial geometry, fibre tension and draw ratio that will yield the desired fibre geometry for a given value of $\gamma^{*}$. It is not clear to us that this must be so or that instability is likely for the reasons given. As already discussed, buckling instability is only to be expected when the fibre tension is negative. On the other hand draw-resonance instability is not really a practical problem and, in any case, is a consequence of a sufficiently large draw ratio (Yarin et al. 1994), which is not restricted to one branch of the $\left(D, \sigma^{*}\right)$ curve, and, in addition, the region of probable instability identified by Griffiths \& Howell (2008) (in which the tension decreases with draw ratio) is in the vicinity of the minimum draw ratio. Noting that the $M \gamma^{*}$ 
contours in $\left(D, \sigma^{*}\right)$ space are parameterised by $\tau_{L}$, equivalently $\alpha_{0}$, our conclusion is that the mathematics indicates that a given geometry can be drawn from two different initial conditions with an appropriate choice of the fibre tension. It is to be expected that the draw parameters will depend on the initial geometry and that one initial geometry will enable smaller draw ratio and fibre tension. The fibre tension $\sigma^{*}$ is a decreasing function of $D$ where the draw ratio is near to its minimum value but, provided $D>\left(\alpha_{0} / \alpha_{L}\right)^{1 / 3}$, there is no real reason to suspect instability. Rather, this is a consequence of change in the initial geometry from which the fibre is drawn. Nevertheless, the higher fibre tension may cause breaking of the fibre and not be appropriate on that account.

In practice fluctuations during fibre drawing of the preform geometry (i.e. $\alpha_{0}$ ), the fibre tension, the draw speed, the temperature and, hence, $M$, or the value of $\gamma^{*}$ which depends on the feed speed (as well as material properties and the slenderness of the geometry) will almost certainly result in modification of the final fibre geometry which is determined by the initial geometry and the draw parameters.

\subsection{Model validation}

The authors are currently engaged in exploring the full experimental implications of the model presented in this work; the results of that ongoing work will be presented in detail elsewhere. It is possible, however, to offer some preliminary evidence that validates our model.

We selected drawing of capillaries from F2 glass, a commercial lead-silicate glass from the Schott Glass Company (http://www.schott.com/advanced_optics/english/ products/optical-materials/optical-glass/optical-glass/index.html). An F2 glass capillary of external diameter $\sim 150 \mu \mathrm{m}\left(R_{F} \sim 75 \mu \mathrm{m}\right)$ is typically drawn from a preform of external diameter $10 \mathrm{~mm}\left(R_{P}=5 \mathrm{~mm}\right)$ and with a central air channel of diameter $2 \mathrm{~mm}\left(\rho_{0}=0.2, S_{0}=25 \pi\left(1-0.2^{2}\right) \approx 75 \mathrm{~mm}^{2}\right)$ using a fibre tension of around $\sigma=0.3 \mathrm{~N}$ and a viscosity in the range $\mu=10^{5}-10^{6} \mathrm{~Pa}$ s. Typical values of the feed speed and the neck-down length are $U_{0}=1.4 \mathrm{~mm} \mathrm{~m}^{-1}$ and $L=4 \mathrm{~cm}$, respectively. Then $D \approx\left(R_{P} / R_{F}\right)^{2} \approx 4400$. The surface tension of $\mathrm{F} 2$ glass has been measured at $\gamma=0.23 \mathrm{~N} \mathrm{~m}^{-1}$ (Boyd et al. 2012).

Running the forward model using $D=4400, \alpha_{0}=\sqrt{2 /(3 \pi)}\left(\rho_{0}=0.2\right)$, for $0 \leqslant \tau_{L} \leqslant$ $\tau_{\max }$, gives the scaled viscosity-modified surface tension and fibre tension, $M \gamma^{*}$ and $M \sigma^{*}$, as functions of $\tau_{L}$ as shown in figure 8 .

In order to determine physical draw parameters from our model solution, we need to obtain dimensional information from the dimensionless parameters $D, \alpha_{0}, M \gamma^{*}$ and $M \sigma^{*}$. The physical fibre tension and surface tension are given by (see (2.3) and (2.7))

$$
\sigma=\frac{6 \hat{\mu} U_{0} S_{0}}{L} M \sigma^{*}, \quad \gamma=\frac{\hat{\mu} U_{0} \sqrt{S_{0}}}{L} M \gamma^{*},
$$

where $\hat{\mu}=\mu_{0} / M$ is the harmonic mean of the viscosity which we here call the effective viscosity. In the case of constant viscosity, $M=1$ and $\mu_{0}=\hat{\mu}$. Since $\gamma$ is a specified physical quantity, we use $(4.15 b)$ to compute $\hat{\mu}$ corresponding to $M \gamma^{*}$, from which we, in turn, compute the effective temperature $T$ via the Vogel-Fulcher-Tamann (VFT) temperature-viscosity relation for F2 glass (K. Richardson, 2012, private communication as part of NSF-DMR \#0807016 'Materials world network in advanced optical glasses for novel optical fibres'):

$$
\log _{10} \hat{\mu}=-2.314+\frac{4065.2}{T-137}
$$




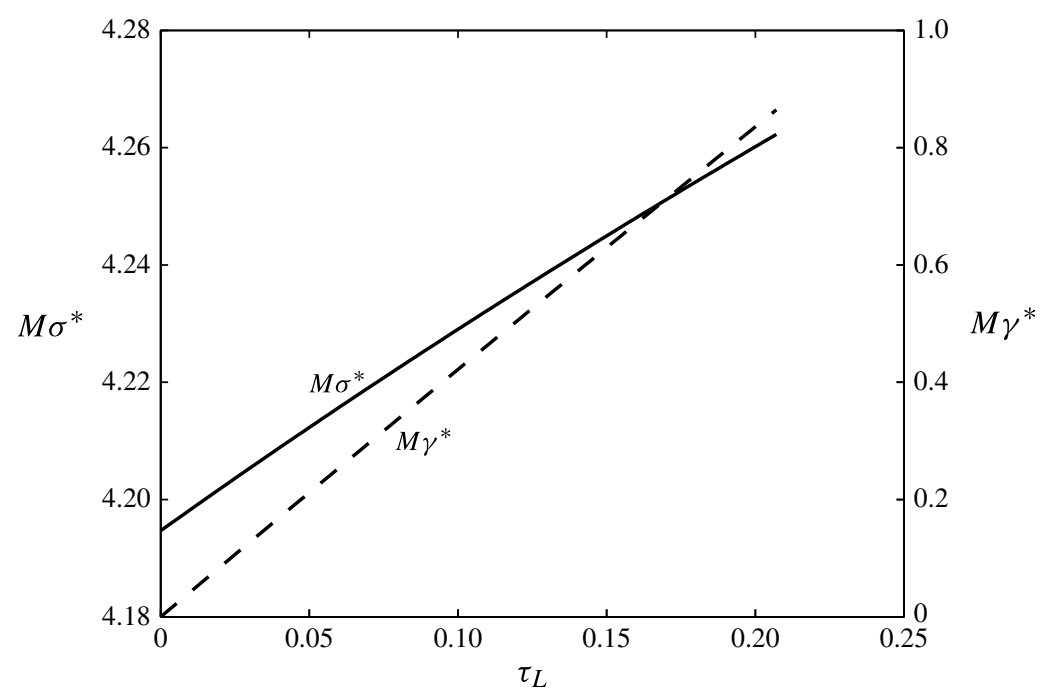

FIGURE 8. Scaled viscosity-modified fibre tension $M \sigma^{*}$ (solid) and surface tension $M \gamma^{*}$ (dashed) versus $\tau_{L}, 0 \leqslant \tau_{L} \leqslant \tau_{\max }, \tau_{\max }=2\left(1 / \sqrt{\pi}-\alpha_{0}\right)$, for $\alpha_{0}=\sqrt{2 /(3 \pi)}\left(\rho_{L}=0.2\right)$.

where $\hat{\mu}$ is the effective viscosity in $\mathrm{Pa} \mathrm{s}$ and $T$ is the effective temperature in ${ }^{\circ} \mathrm{C}$. Figure $9(a-e)$ features plots of the physical fibre tension $\sigma$, effective viscosity $\hat{\mu}$, fibre external radius $R_{F}$, internal radius as a fraction of the external radius $\rho_{L}$, internal radius $\rho_{L} R_{F}$ and effective temperature. For $\tau_{L} \approx 0.04$, i.e. $R_{F} \approx 74.8 \mu \mathrm{m}$, we see that $\sigma \sim 30 \mathrm{~N}, \hat{\mu} \sim 2.7 \times 10^{5} \mathrm{~Pa}$ s and $T \sim 662{ }^{\circ} \mathrm{C}$, in agreement with the experimental data supplied. This gives us confidence in the validity of our model.

Finally, we note that in general $R_{P}=5 \mathrm{~mm}$ and $0<\rho_{0}<0.6$ so that $0.18<\epsilon<0.23$ if $L=4 \mathrm{~cm}$. This justifies our primary assumption of small $\epsilon$.

\section{The question of uniqueness}

The two case studies lead naturally to an important question concerning the uniqueness of solutions to the inverse problem. For the annular tube example, figure $6(a)$ shows that a given fibre geometry can be drawn from two different initial geometries. Is this a feature that is specific to this particular example or should one expect it when drawing a fibre of general shape?

While we cannot give a definitive answer to this, important insights can be gained by contrasting figure 6 (pertaining to the concentric annulus case study) with figure 10 which shows the same analysis of the inverse problem carried out for the first case study of $\S 3$ putting $N=10$, for which the initial value of $\rho$ given in table 1 of Crowdy (2003) is $\rho_{0}=0.81$. For comparison with the annulus, the same value $\rho_{L}=0.2$ is used here as in figure 6. Figures $10(a)$ and $10(b)$ show contours of $M \gamma^{*}$ and $M \sigma^{*}$ in $\left(D, \tau_{L}\right)$ space, respectively.

The most obvious difference between the two case studies is that, while the solution for the axisymmetric annular cross-section can be obtained very far backwards in reduced time, to $\rho_{0} \rightarrow 1$ when the inner and outer boundaries of the cross-section meet and the preform radius becomes infinite, this is not possible for the solution for the non-axisymmetric cross-section of $\S 3$. When $\rho(\tau)$ exceeds the value $\rho_{0}$, which for finite $N$ is necessarily less than unity, the boundaries of the sintering cylinders 

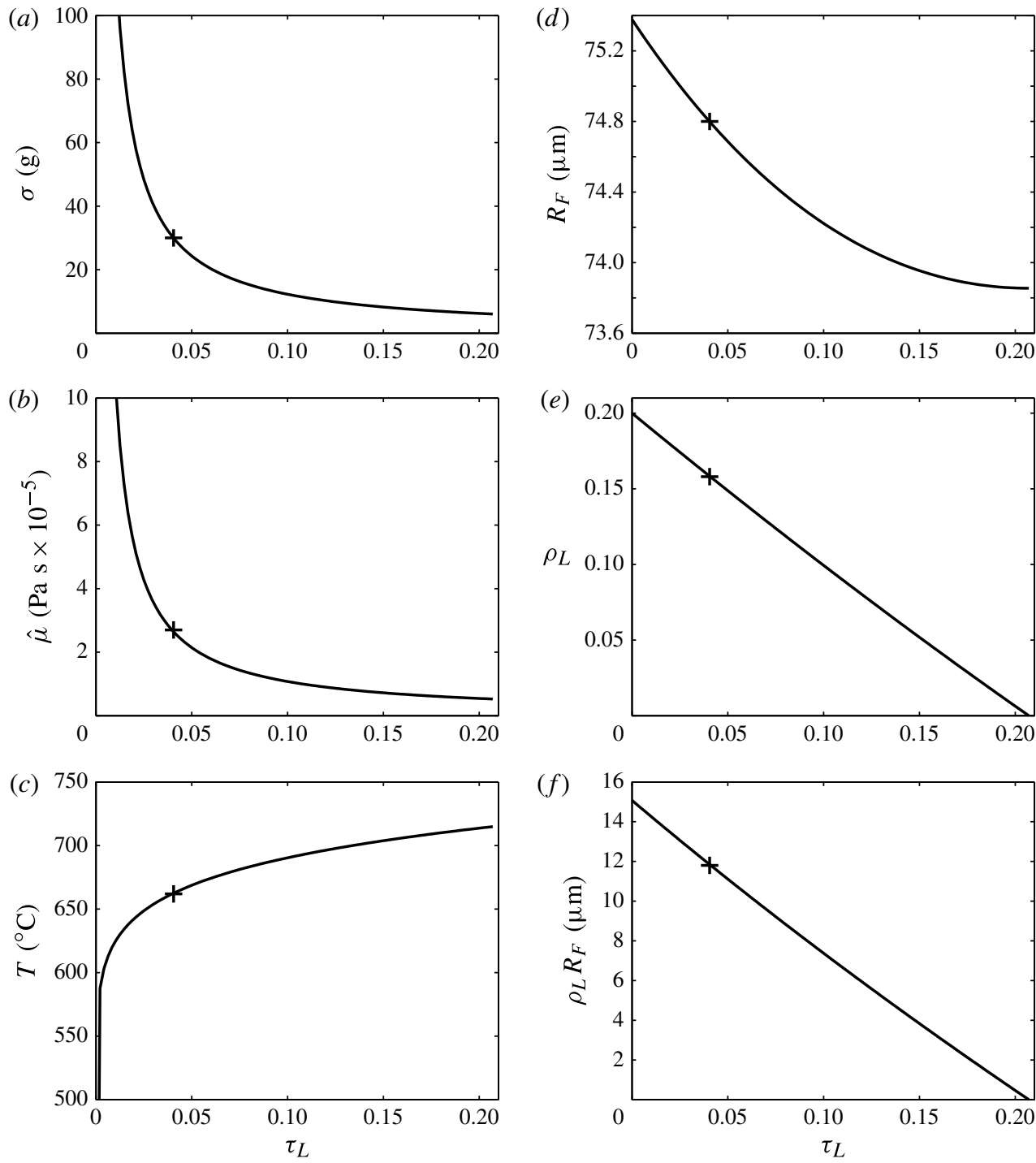

FIGURE 9. Drawing of a glass circular-tube preform with external diameter $10 \mathrm{~mm}$ (radius $\left.R_{P}=5 \mathrm{~mm}\right)$ and internal diameter $2 \mathrm{~mm}(\rho=0.2)$. The glass has surface tension $\gamma=$ $0.23 \mathrm{~N} \mathrm{~m}^{-1}$, the feed speed is $U_{0}=1.4 \mathrm{~mm} \mathrm{~m}^{-1}$ and the draw ratio is $D=4400$. The neck-down region is assumed to have length $L=4 \mathrm{~cm}$. Plotted as functions of the scaled deformation time $\tau_{L}$, which determines the fibre geometry, are: $(a)$ the fibre tension $\sigma$, (b) the effective viscosity $\hat{\mu},(c)$ the effective temperature $T,(d)$ the external fibre radius $R_{F}$, (e) the aspect ratio $\rho_{L}$ of the annular cross-section of the fibre and $(f)$ the internal fibre radius $\rho_{L} R_{F}$. On each plot ' + ' marks the point $\tau_{L}=0.0405$ at which $\sigma=30 \mathrm{~g}, R_{F}=$ $74.8 \mu \mathrm{m}, \hat{\mu}=2.7 \times 10^{5} \mathrm{~Pa} \mathrm{~s}, \rho_{L}=0.158, T=662^{\circ} \mathrm{C}, \rho_{L} R_{F}=11.8 \mu \mathrm{m}$.

intersect and the solution becomes non-physical. The requirement that the solution describe a physically achievable cross-section thus limits the reduced time $\tau_{L}$ over which sintering can occur to the time it takes $\rho(\tau)$ to evolve from $\rho_{0}$ to $\rho_{L}$. This is shown in figure 10 which is plotted over the maximum possible range of $\tau_{L}$. 

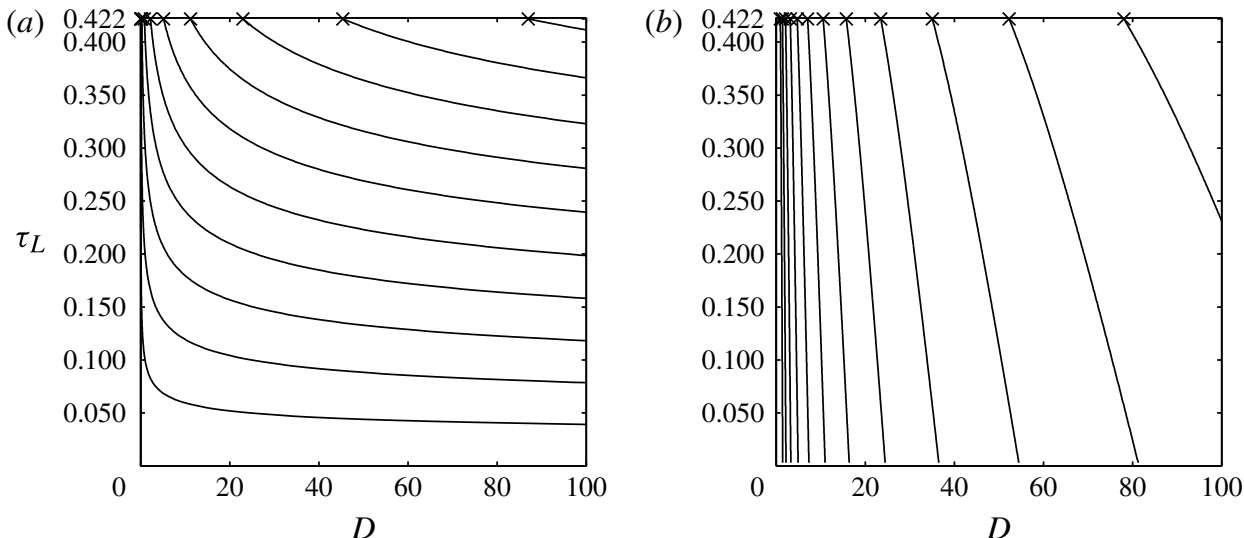

FIGURE 10. Solutions of the inverse problem for the example described in $\S 3$, for $N=10$ and $\rho_{L}=0.2$, shown as contours of $M \gamma^{*}\left(D, \tau_{L}\right)$ and $M \sigma^{*}\left(D, \tau_{L}\right):(a) M \gamma^{*}=$ $0.1,0.2, \ldots, 1.0$ (bottom to top) and (b) $M \sigma^{*}=0.2,0.4, \ldots, 2.4$ (left to right). The contours extend to only $\tau_{L}=0.422$ (endpoints marked $\times$ ), above which the value of $\rho$ for the initial configuration exceeds 0.81 and the map is no longer univalent. For a given geometry, there is only one initial condition that will yield the desired final configuration. This may be contrasted to figure 6 .

This has consequences for the matter of non-uniqueness. It can be seen by comparing figures 6 and 10 that, while a given fibre geometry may be drawn from two different initial geometries for the annulus, this is not so for the sintering circles; for a given choice of $D$ and $M \gamma^{*}$, there is at most one initial configuration. We return to this issue again in the Discussion section.

\section{Ill-posedness of the inverse problem}

Finally, it turns out that the two case studies can also be used to directly illustrate a further difficulty inherent to the inverse problem. Figure 11 shows cross-sections of two draws with different initial configurations that lead to what are arguably indistinguishable final configurations under the same experimental parameters; figure 12 shows three-dimensional diagrams of the corresponding fibres. Since holes shrink and close over time this situation of virtually indistinguishable end-states could even occur with initial configurations having completely different connectivity. This ill-posedness of the inverse surface-tension-driven planar-flow problem has been recognised by others, for example Yarin (1995) and Griffiths \& Howell (2008). The implications of this for computing an initial preform for a desired fibre design are discussed further in the next section.

\section{Discussion}

A principal contribution of this paper has been to highlight the importance of the reduced time $\tau$ as a natural parameter and the significance of the function $H(\tau)$ defined in (2.18) which plays a key role in coupling the surface-tension-driven cross-plane evolution with the axial stretching. The significance of the function $H(\tau)$, and the usefulness of the $\tau$ variable, appears to have been missed by previous investigators. 
(a)

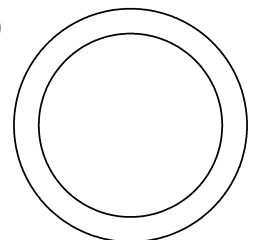

$\tau_{L}=0.30$

(b)

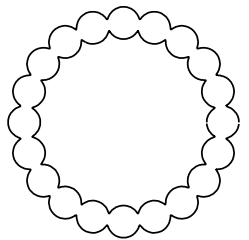

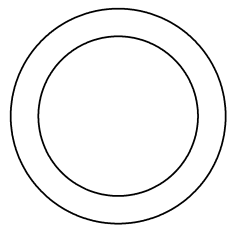

$\tau_{L}=0.25$

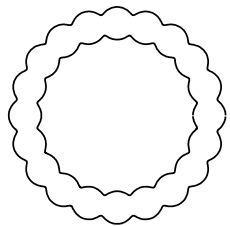

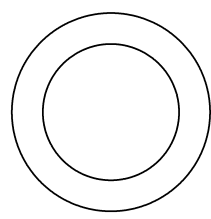

$\tau_{L}=0.20$

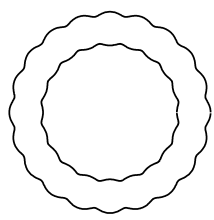

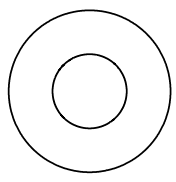

$\tau_{L}=0$

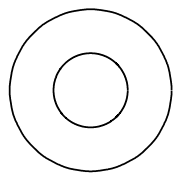

FIgURE 11. Cross-sections of $(a)$ an annulus and $(b) N=20$ sintering circles using the solution from $\S 3.1$. Each cross-section is labelled with $\tau_{L}$, the reduced time interval from that cross-section to the final configuration on the right. Both examples are for $D=400, M \gamma^{*}=0.9$, and $M \sigma^{*}=3.2$. The final configurations, while different, are visually indistinguishable.

(a)

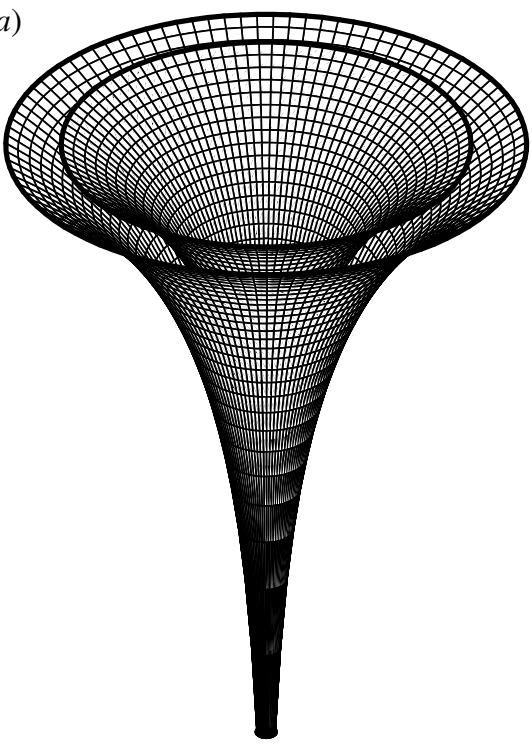

(b)

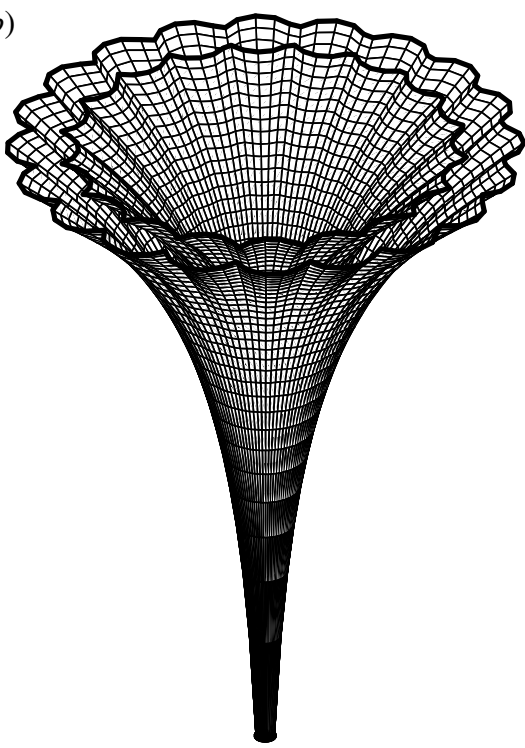

FIGURE 12. Three-dimensional diagram of the two draws from figure 11 , with $M=1$ $\left(\mu^{*}=1\right)$. The transverse dimensions have been stretched relative to the axial dimension.

The formulation makes no assumptions on the cross-plane geometry and this versatility in the approach clearly has ramifications for its use in a broad spectrum of fibre drawing applications.

For given initial geometry and draw parameters - that is, the forward problem there is a unique outcome. The inverse problem is of greater practical interest, namely determining the initial geometry and draw parameters to achieve a desired outcome. There are multiple options for drawing of a given fibre: many practical factors such as 
material properties, temperature control in the draw tower, the temperature dependence of the viscosity, heat transfer effects, channel pressurisation all enter the analysis of which of these are practically achievable. The model considered here is, in a sense, the most basic one in that it incorporates only the interaction of axial tension with surface tension. The temperature dependence of viscosity can be taken into account by use of the harmonic mean of the viscosity, as we have shown, but, importantly, the model gives the final fibre geometry for a given preform, draw ratio and surface tension, without any reference to the viscosity, equivalently temperature, so that where the fibre tension is measured, knowledge of the glass temperature profile is not required.

This paper has demonstrated that the $\tau$ variable is a natural one for the modelling of fibre drawing in the absence of internal channel pressurisation in that, procedurally, it allows a decoupling of the cross-plane and axial problems and, in addition, provides an explicit form for all relevant axially varying quantities. When internal pressurisation is introduced, at first sight these advantages appear to vanish. Nevertheless, we have found that when the channel pressurisation problem is reformulated using another natural independent variable - different from $\tau$, but closely related to it - all the same advantages of the zero-pressurisation model given here are restored. Full details will be presented in a forthcoming article.

A mechanism sometimes used for control of the fibre draw process, that we have not yet considered and which remains for future investigation, is rotation of the fibre during drawing so that the resulting fibre has a twisted geometry (Voyce, Fitt \& Monro 2004, 2008).

The two case studies presented in the current paper have shown that the solution to the inverse problem is not necessarily unique, at least for the case of an annular capillary tube (case study 2). However a comparison with case study 1 shows that the same feature of non-uniqueness is absent for the geometrically non-trivial example given there. Based on this evidence we conjecture that the non-uniqueness in the special concentric annulus example is not generic and should not be of particular concern in the drawing of a fibre of general shape.

A natural suggestion for solving the inverse problem is to reverse time and compute a solution to the backwards-time problem to get predictions for appropriate initial profiles giving rise to desired end-state profiles. Indeed, this is the strategy suggested and adopted by previous authors (Griffiths \& Howell 2007, 2008) in studying the Vello process for drawing glass tubing. Those authors also recognised that running the transverse-flow problem backwards in time is inherently unstable since surface tension in forward time minimises surface energy and serves to iron out ripples to produce an interface with constant curvature; in reverse time this process is reversed and surface energy is injected into the interface implying that even small disturbances, or numerical noise in a computational scheme, will be susceptible to amplification. Figure 11, based on our two case study examples, shows precisely this process in action. This ill-posedness suggests it is important to search for strategic methods to solve the backwards-time problem in a constrained manner so that the possible outcomes for initial profiles are consistent with those that are admissible based on other independent considerations. Griffiths \& Howell (2008) succeed in this endeavour by noting the surprising fact that their asymptotic approach to solving the transverse-flow problem also serves to filter out the growth of extraneous modes in backwards time that would, ordinarily, pollute the backwards-time calculation (see also Griffiths \& Howell 2009). Similarly, Yarin (1995) recognised that truncation of a Fourier-series solution of the surface-tension-driven flow in almost circular geometries enabled solution of the inverse problem. 
Our primary interest is in modelling the drawing of more geometrically complicated fibres such as microstructured optical fibres, or MOFs, which often consist of many channels extending along their length. In a companion paper (Buchak, Crowdy \& Stokes 2014), we present details of a model of MOF drawing based on the new formulation given here. We believe that such reduced models will be important in practice and will provide a valuable tool for experimentalists that will require a relatively small amount of computational effort but that will be more generally applicable and more accurate than empirically-based adaptations of the model of Fitt et al. (2002) such as proposed in Kostecki et al. (2014). We note that some three-dimensional numerical simulations of fibre drawing have been performed for MOFs having 2-4 air channels (Xue et al. 2005a,b,c), but this is prohibitively difficult and computationally expensive for the complex structures typically seen in MOFs (10-60 channels, or more) thereby necessitating the need for more strategic mathematical modelling. The method presented in this paper is capable of efficient handling of complex structures and yields fundamental understanding not readily acquired through numerical simulation.

Finally we note that should inertia be important for some application, this can be incorporated into the model with the effect that the differential equation for the one-dimensional stretching flow becomes nonlinear and must be solved numerically. Although we can no longer write down the solution in terms of the function $H(\tau)$, the stretching problem is still coupled to the transverse-flow problem by the total boundary length and, for a given preform and surface tension, the final fibre geometry is still determined by the draw ratio and the fibre tension.

\section{Acknowledgements}

This research is supported by a Research Grant from the Leverhulme Trust in the United Kingdom and grant DP130101541 from the Australian Research Council. It was in part performed at the Optofab node of the Australian National Fabrication Facility utilizing Commonwealth and SA State Government funding.

\section{Appendix A}

Section 3.1 involves use of a function $I(\zeta, \tau)$ given in Crowdy (2003). For completeness, and to correct a minor typographic error in the original paper, we include it here:

$$
I(\zeta, \tau)=I^{+}(\zeta, \tau)-I^{-}(\zeta, \tau)+C(\tau)
$$

where

$$
\begin{gathered}
I^{+}(\zeta, \tau)=\frac{1}{4 \pi \mathrm{i}} \oint_{|\eta|=1} \frac{\mathrm{d} \eta}{\eta}\left(1-2 \frac{\zeta}{\eta} \frac{P_{1}^{\prime}(\zeta / \eta, \rho)}{P_{1}(\zeta / \eta, \rho)}\right) \frac{1}{\left|z^{\prime}(\eta, \tau)\right|} \\
I^{-}(\zeta, \tau)=\frac{1}{4 \pi \mathrm{i}} \oint_{|\eta|=\rho} \frac{\mathrm{d} \eta}{\eta}\left(1-2 \frac{\zeta}{\eta} \frac{P_{1}^{\prime}(\zeta / \eta, \rho)}{P_{1}(\zeta / \eta, \rho)}\right)\left(-\frac{1}{\rho\left|z^{\prime}(\eta, \tau)\right|}-\frac{2}{\rho} \frac{\mathrm{d} \rho}{\mathrm{d} \tau}\right), \\
C(\tau)=-\frac{1}{4 \pi \mathrm{i}} \oint_{|\eta|=\rho} \frac{\mathrm{d} \eta}{\eta}\left(-\frac{1}{\rho\left|z^{\prime}(\eta, \tau)\right|}-\frac{2}{\rho} \frac{\mathrm{d} \rho}{\mathrm{d} \tau}\right) .
\end{gathered}
$$

Note that in these integrands $z^{\prime}$ is evaluated at $\eta$, not at $\zeta$ as indicated in Crowdy (2003).

In order to compute the function $H(\tau)$ for the first case study we need to compute the total perimeter $\tilde{\Gamma}(\tau)$. As seen from (3.3) this requires knowledge of the 
function $z^{\prime}(\zeta)$. From $(3.1 a)$ it can be shown that

$$
\frac{\zeta z^{\prime}(\zeta, \tau)}{z(\zeta, \tau)}=\frac{\zeta f^{\prime}(\zeta, \tau)}{f(\zeta, \tau)}=1+K_{N}\left(\zeta \rho^{2 / N} a^{-1}, \rho\right)-K_{N}\left(\zeta a^{-1}, \rho\right),
$$

with the function $K_{N}(\zeta, \rho)$ defined by the infinite sum

$$
K_{N}(\zeta, \rho) \equiv \frac{\zeta P_{N}^{\prime}(\zeta, \rho)}{P_{N}(\zeta, \rho)}=-\frac{N \zeta^{N}}{1-\zeta^{N}}-\sum_{k=1}^{\infty} \frac{N \rho^{2 k N} \zeta^{N}}{1-\rho^{2 k N} \zeta^{N}}+\sum_{k=1}^{\infty} \frac{N \rho^{2 k N} / \zeta^{N}}{1-\rho^{2 k N} / \zeta^{N}}
$$

The perimeter $\tilde{\Gamma}(\tau)$, and hence $H(\tau)$, can be readily computed on use of these formulae.

\section{REFERENCES}

Boyd, K., Ebendorff-Heidepriem, H., Monro, T. M. \& Munch, J. 2012 Surface tension and viscosity measurement of optical glasses using a scanning $\mathrm{CO}_{2}$ laser. Opt. Mater. Express 2 (8), 1101-1110.

Buchak, P., Crowdy, D. G. \& Stokes, Y. 2014 Elliptical pore regularization of the inverse problem for microstructured optical fibre fabrication. J. Fluid Mech. (submitted).

Chen, Y. \& BIRKs, T. A. 2013 Predicting hole sizes after fibre drawing without knowing the viscosity. Opt. Mater. Express 3 (3), 346-356.

CROWDY, D. G. 2003 Viscous sintering of unimodal and bimodal cylindrical packings with shrinking pores. Eur. J. Appl. Maths 14, 421-445.

Crowdy, D. G. \& TANVEeR, S. $1998 a$ A theory of exact solutions for plane viscous blobs. J. Nonlinear Sci. 8 (3), 261-279.

Crowdy, D. G. \& TANVEeR, S. $1998 b$ A theory of exact solutions for annular viscous blobs. J. Nonlinear Sci. 8 (4), 375-400.

Cummings, L. J. \& Howell, P. D. 1999 On the evolution of non-axisymmetric viscous fibres with surface tension, inertia and gravity. J. Fluid Mech. 389, 361-389.

Cummings, L. J., Howison, S. D. \& King, J. R. 1997 Conserved quantities in Stokes flow with free surfaces. Phys. Fluids 9, 477-480.

Denn, M. M. 1980 Continuous drawing of liquids to form fibres. Annu. Rev. Fluid Mech. 12, 365-387.

DeWynne, J. N., Howell, P. D. \& Wilmott, P. 1994 Slender viscous fibres with inertia and gravity. Q. J. Mech. Appl. Maths 47, 541-555.

Fitt, A. D., Furusawa, K., Monro, T. M., Please, C. P. \& Richardson, D. A. 2002 The mathematical modelling of capillary drawing for holey fibre manufacture. J. Engng Maths 43, 201-227.

Gospodinov, P. \& YARIN, A. L. 1997 Draw resonance of optical microcapillaries in non-isothermal drawing. Intl J. Multiphase Flow 23, 967-976.

GRIFFITHS, I. M. \& Howell, P. D. 2007 The surface-tension-driven evolution of a two-dimensional annular viscous tube. J. Fluid Mech. 593, 181-208.

GRIfFiths, I. M. \& Howell, P. D. 2008 Mathematical modelling of non-axisymmetric capillary tube drawing. J. Fluid Mech. 605, 181-206.

Griffiths, I. M. \& Howell, P. D. 2009 The surface-tension-driven retraction of a viscida. SIAM J. Appl. Maths 70 (5), 1453-1487.

Hopper, R. W. 1990 Plane Stokes flow driven by capillarity on a free surface. J. Fluid Mech. 213, 349-375.

Howell, P. D. 1994 Extensional thin layer flows. PhD thesis, University of Oxford.

KAYE, A. 1990 Convected coordinates and elongational flow. J. Non-Newtonian Fluid Mech. 40, 55-77. 
Knight, J. C. 2003 Photonic crystal fibres. Nature 424, 847-851.

Kostecki, R., Ebendorff-Heidepriem, H., Warren-Smith, S. C. \& Monro, T. M. 2014 Predicting the drawing conditions for microstructured optical fibre fabrication. Opt. Mater. Express 4, 29-40.

LANGlois, W. 1964 Slow Viscous Flows. Macmillan.

Matovich, M. A. \& Pearson, J. R. A. 1969 Spinning a molten threadline. Ind. Engng Chem. Fundam. 8, 512-520.

Monro, T. M. \& Ebendorff-Heidepriem, H. 2006 Progress in microstructured optical fibres. Annu. Rev. Mater. Res. 36, 467-495.

Muskhelishvili, N. I. 1977 Some Basic Problems in the Mathematical Theory of Elasticity. Springer.

Pearson, J. R. A. \& Matovich, M. A. 1969 Spinning a molten threadline; stability. Ind. Engng Chem. Fundam. 8, 605-609.

RichARDSON, S. 1992 Two-dimensional slow viscous flows with time dependent free boundaries driven by surface tension. Eur. J. Appl. Maths 3, 198-207.

RichARDSON, S. 2000 Plane Stokes flow with time-dependent free boundaries in which the fluid occupies a doubly connected region. Eur. J. Appl. Maths 11, 249-269.

Scheid, B., Quiligotti, S., Tranh, B., Gy, R. \& Stone, H. A. 2010 On the (de)stabilization of draw resonance due to cooling. J. Fluid Mech. 636, 155-176.

Stokes, Y. M., Tuck, E. O. \& Schwartz, L. W. 2000 Extensional fall of a very viscous fluid drop. Q. J. Mech. Appl. Maths 53, 565-582.

Taroni, M., Breward, C. J. W., Cummings, L. J. \& Griffiths, I. M. 2013 Asymptotic solutions of glass temperature profiles during steady optical fibre drawing. J. Engng Maths 80, 1-20.

Tchavdarov, B., Yarin, A. L. \& Radev, S. 1993 Buckling of thin liquid jets. J. Fluid Mech. 253, 593-615.

VAN DE Vorst, G. A. L. \& Mattheij, R. M. M. 1995 A BEM-BDF scheme for curvature driven moving Stokes flows. J. Comput. Phys. 120, 1-14.

Voyce, C. J., Fitt, A. D. \& Monro, T. M. 2004 Mathematical model of the spinning of microstructured fibres. Opt. Express 12 (23), 5810-5820.

Voyce, C. J., FitT, A. D. \& Monro, T. M. 2008 The mathematical modelling of rotating capillary tubes for holey-fibre manufacture. J. Engng Maths 60, 69-87.

Wilson, S. D. R. 1988 The slow dripping of a viscous fluid. J. Fluid Mech. 190, 561-570.

Wylie, J. J., HuAng, H. \& MiURA, R. M. 2007 Thermal instability in drawing viscous threads. J. Fluid Mech. 570, 1-16.

Xue, S. C., Large, M. C. J., Barton, G. W., Tanner, R. I., Poladian, L. \& Lwin, R. $2005 a$ Role of material properties and drawing conditions in the fabrication of microstructured optical fibres. J. Lightwave Technol. 24, 853-860.

Xue, S. C., Tanner, R. I., Barton, G. W., Lwin, R., Large, M. C. J. \& Poladian, L. $2005 b$ Fabrication of microstructured optical fibres - part I: problem formulation and numerical modelling of transient draw process. J. Lightwave Technol. 23, 2245-2254.

Xue, S. C., Tanner, R. I., Barton, G. W., Lwin, R., Large, M. C. J. \& Poladian, L. 2005c Fabrication of microstructured optical fibres - part II: numerical modelling of steady-state draw process. J. Lightwave Technol. 23, 2255-2266.

YARIN, A. L. 1993 Free Liquid Jets and Films: Hydrodynamics and Rheology. Longman Scientific $\&$ Technical and Wiley \& Sons.

YARIN, A. L. 1995 Surface-tension-driven flows at low Reynolds number arising in optoelectronic technology. J. Fluid Mech. 286, 173-200.

YARIN, A. L., Gospodinov, P. \& Roussinov, V. I. 1994 Stability loss and sensitivity in hollow fibre drawing. Phys. Fluids 6, 1454-1463.

YARin, A., Rusinov, V. I., Gospodinov, P. \& RADEV, ST. 1989 Quasi one-dimensional model of drawing of glass micro capillaries and approximate solutions. Theor. Appl. Mech. 20 (3), 55-62. 